\title{
The Dirichlet-to-Neumann operator for divergence form problems
}

\author{
A. F. M. ter Elst $^{1}$ - G. Gordon ${ }^{1} \cdot$ M. Waurick ${ }^{2}$
}

Received: 26 October 2017 / Accepted: 24 June 2018 / Published online: 4 July 2018

(c) Fondazione Annali di Matematica Pura ed Applicata and Springer-Verlag GmbH Germany, part of Springer Nature 2018

\begin{abstract}
We present a way of defining the Dirichlet-to-Neumann operator on general Hilbert spaces using a pair of operators for which each one's adjoint is formally the negative of the other. In particular, we define an abstract analogue of trace spaces and are able to give meaning to the Dirichlet-to-Neumann operator of divergence form operators perturbed by a bounded potential in cases where the boundary of the underlying domain does not allow for a welldefined trace. Moreover, a representation of the Dirichlet-to-Neumann operator as a first-order system of partial differential operators is provided. Using this representation, we address convergence of the Dirichlet-to-Neumann operators in the case that the appropriate reciprocals of the leading coefficients converge in the weak operator topology. We also provide some extensions to the case where the bounded potential is not coercive and consider resolvent convergence.
\end{abstract}

Keywords Dirichlet-to-Neumann operator · Resolvent convergence · Continuous dependence on the coefficients

Mathematics Subject Classification 35F45 · 46E35 · 47A07

\section{Introduction}

In the theory of elliptic partial differential operators, the Dirichlet-to-Neumann operator is a central object of study. In recent years, it has attracted a lot of attention and triggered

A. F. M. ter Elst

terelst@math.auckland.ac.nz

G. Gordon

g.gordon@auckland.ac.nz

M. Waurick

marcus.waurick@strath.ac.uk

1 Department of Mathematics, University of Auckland, Auckland 1142, New Zealand

2 Department of Mathematics and Statistics, University of Strathclyde, Livingstone Tower, 26 Richmond Street, Glasgow G1 1XH, UK 
profound research in many directions. In particular, we mention applications of the form method, relations to the extension theory of symmetric operators as well as the intimate connection to the Calderón problem, see, for instance, the references in [6].

The Dirichlet-to-Neumann operator relates Dirichlet boundary data to the corresponding Neumann boundary data of solutions to a partial differential equation. As an introduction, we provide a definition for the Dirichlet-to-Neumann operator in its arguably simplest form.

Let $\Omega \subset \mathbb{R}^{d}$ be a bounded domain with smooth boundary $\Gamma=\partial \Omega$ and where $d \geqslant 2$. Note that in this case, the trace map $\operatorname{Tr}$ from $H^{1}(\Omega)$ into $H^{1 / 2}(\Gamma)$ is a well-defined, surjective and continuous operator. Let $\varphi \in H^{1 / 2}(\Gamma)$, and let $u \in H^{1}(\Omega)$ be the solution of the boundary value problem

$$
-\Delta u=0 \text { weakly on } \Omega \text { and } \operatorname{Tr} u=\varphi
$$

The Dirichlet-to-Neumann operator $\Lambda$ assigns to $\varphi$ the normal derivative of $u$, that is, $\Lambda \varphi=$ $\partial_{\nu} u \in H^{-1 / 2}(\Gamma)$.

We can also consider the part of $\Lambda$ in $L_{2}(\Gamma)$. If we call this restriction $\Lambda_{L_{2}(\Gamma)}$, then $\Lambda_{L_{2}(\Gamma)}$ is an unbounded operator in $L_{2}(\Gamma)$ such that for all $\varphi, \psi \in L_{2}(\Gamma)$ it follows that $\varphi \in \operatorname{dom}\left(\Lambda_{L_{2}(\Gamma)}\right)$ and $\Lambda_{L_{2}(\Gamma)} \varphi=\psi$ if and only if there exists a $u \in H^{1}(\Omega)$ such that $-\Delta u=0$ weakly on $\Omega$, $\operatorname{Tr} u=\varphi$ and $\psi=\partial_{\nu} u$. A problem with the above descriptions is that they only make sense if the boundary of $\Omega$ is sufficiently smooth. We may also refer to [2] for a variant of the Dirichlet-to-Neumann operator for domains with a rough boundary that has finite $(d-1)$-dimensional Hausdorff measure. If, however, $\Omega$ has for example a fractal boundary with infinite $(d-1)$-dimensional Hausdorff measure, then in [2] there is no notion of the Dirichlet-to-Neumann operator at hand simply because there is no appropriate notion of a trace. Using the concepts developed in [13] (with extensions in [12] and [16]), we are able to provide a substitute for the space $H^{1 / 2}(\Gamma)$. We note here that this 'trace-free' concept has proven to be useful for dealing with boundary value problems on domains with rough boundary, see [11].

The substitute for the space $H^{1 / 2}(\Gamma)$ is a variant of 1 -harmonic functions in $\Omega$. This removes the need for function evaluation at the boundary. For the definition of this substitute of $H^{1 / 2}(\Gamma)$, the only concept that we use, if we relate our findings to the Laplacian, is that the matrix $\left(\begin{array}{c}0 \\ \operatorname{grad} \\ \text { div }\end{array}\right)$ is skew-symmetric on the space of infinite differentiable functions with compact support, see Example 2.3. Thus, without further effort, our results directly apply to similar problems involving the equations of linearized elasticity or the full three-dimensional system of static Maxwell's equations. More generally, our methods apply to the covariant derivative defined on suitable $L_{2}$-tensor fields and a formal skew-adjoint.

As our central object of study, we shall deviate from the classical elliptic partial differential operator $-\Delta$ discussed above and treat abstract divergence form operators of the form

$$
-D a G+m,
$$

where $a$ and $m$ are bounded coercive operators (called coefficients) and $D$ and $G$ are densely defined, closed, unbounded operators in Hilbert spaces $H_{1}$ and $H_{0}$ with the property $-D^{*} \subset$ $G$, like div and grad.

If $\operatorname{dom}(G)$, endowed with the graph norm, embeds compactly into $H_{0}$, we will also address the concept of continuous dependence of the Dirichlet-to-Neumann operator associated with (1) on the bounded coefficients $a$ and $m$ under the weak operator topology. This result has applications in homogenization problems, see [15] and [18] Section 5.5. Moreover, it complements the study of continuous dependence of the Dirichlet-to-Neumann operator on its coefficients in [4], where the authors focus on possible non-coercive cases and convergence 
of the principal coefficients in $L_{\infty}(\Omega)$. In order to prove convergence results, we derive a reformulation of the Dirichlet-to-Neumann operator as a system of two first-order partial differential equations, similar to [5].

In the present work, we also consider removing the coercivity condition on $m$. That is to say, we define the abstract analogue of the Dirichlet-to-Neumann graph with $m$ being possibly not coercive. We note here that these results are the abstract counterpart of results developed in [6] and [4]. In the case that the potentials $m$ are not coercive, we consider resolvent convergence for Dirichlet-to-Neumann operators. Under different assumptions, convergence of Neumann-to-Dirichlet operators was obtained in [14].

We mention here that a possible nonlinear variant of the Dirichlet-to-Neumann operator, where the coercive operator $a$ is replaced by a (strictly) maximal monotone relation, can be discussed using the results of [17]. This, however, is beyond the scope of the present manuscript and will be addressed in future work.

We briefly comment on the organization of the paper. In Sect. 2, we provide the basic functional analytic setting and recall some notions and results of [12,13] and [16]. We then state the definition of the Dirichlet-to-Neumann operator in the abstract setting discussed above. We also provide an extensive example that justifies this abstraction by relating it to the classical formulation of the Dirichlet-to-Neumann operator. In Sect. 3, we give a representation formula for the Dirichlet-to-Neumann operator in terms of a first-order system and show that this operator is $\mathrm{m}$-sectorial, provided both $m$ and $a$ are coercive. For this, we use a representation result for operators given via forms, see [3]. In Sect. 4, we prove resolvent convergence of the Dirichlet-to-Neumann operators when the coefficients converge in an appropriate weak operator topology. Under some additional hypotheses, we also obtain in Theorem 4.2 uniform convergence even though the coefficients converge in the weak operator topology only. In Sect. 5, we consider the non-coercive case and discuss the domain and multivalued parts of the Dirichlet-to-Neumann graph when $m$ is merely assumed to be a bounded operator, that is, not necessarily coercive. Moreover, we also prove a convergence theorem for the non-coercive case in Sect. 6. We conclude with two more examples in Sect. 7.

\section{The Dirichlet-to-Neumann operator and boundary spaces}

We start with a description of boundary data spaces as in [13] Subsection 5.2. Throughout this paper, fix Hilbert spaces $H_{0}$ and $H_{1}$. Further, let $G$ be an operator in $H_{0}$ with values in $H_{1}$ and let $D$ be an operator in $H_{1}$ with values in $H_{0}$. We assume throughout that both $G$ and $D$ are densely defined and closed, and that $-G^{*} \subset D$. We define $\stackrel{\circ}{D}=-G^{*}$ and $\stackrel{\circ}{G}=-D^{*}$.

Note that

$$
(\stackrel{\circ}{G} u, q)_{H_{1}}=-(u, \stackrel{\circ}{D} q)_{H_{0}}
$$

for all $u \in \operatorname{dom}(\stackrel{\circ}{G})$ and $q \in \operatorname{dom}(\stackrel{D}{D})$. Equivalently, the matrix

$$
\left(\begin{array}{cc}
0 & \stackrel{\circ}{D} \\
\stackrel{\circ}{G} & 0
\end{array}\right)
$$

with dense domain $\operatorname{dom}(\stackrel{\circ}{G}) \times \operatorname{dom}(\stackrel{D}{D})$ is skew-symmetric in $H_{0} \times H_{1}$.

Remark 2.1 Note that $\stackrel{\circ}{G}=-D^{*} \subset-\left(-G^{*}\right)^{*}=\bar{G}=G$. So one can simultaneously swap $H_{0}$ with $H_{1}$ and $D$ with $G$. 
Example 2.2 All examples in this paper are of the following type. Let $H_{0}$ and $H_{1}$ be Hilbert spaces. Consider dense subspaces $\operatorname{dom}(\widehat{G}) \subset H_{0}$ and $\operatorname{dom}(\widehat{D}) \subset H_{1}$. Let $\widehat{G}: \operatorname{dom}(\widehat{G}) \rightarrow$ $H_{1}$ and $\widehat{D}: \operatorname{dom}(\widehat{D}) \rightarrow H_{0}$ be two operators such that

$$
(\widehat{G} u, q)_{H_{1}}=-(u, \widehat{D} q)_{H_{0}}
$$

for all $u \in \operatorname{dom}(\widehat{G})$ and $q \in \operatorname{dom}(\widehat{D})$. Equivalently, the matrix

$$
\left(\begin{array}{cc}
0 & \widehat{D} \\
\widehat{G} & 0
\end{array}\right)
$$

with dense domain $\operatorname{dom}(\widehat{G}) \times \operatorname{dom}(\widehat{D})$ is skew-symmetric in $H_{0} \times H_{1}$.

Then, $\widehat{G} \subset-(\widehat{D})^{*}$ and $\widehat{D} \subset-(\widehat{G})^{*}$, so both $\widehat{G}$ and $\widehat{D}$ are closable. Let $\stackrel{\circ}{G}$ and $\stackrel{\circ}{D}$ denote the closures. Define $G=-(\stackrel{\circ}{D})^{*}$ and $D=-(\stackrel{\circ}{G})^{*}$. Since $\stackrel{\circ}{D}$ and $\stackrel{\circ}{G}$ are closed, therefore closable, it follows that $G$ and $D$ are densely defined. Obviously, both $G$ and $D$

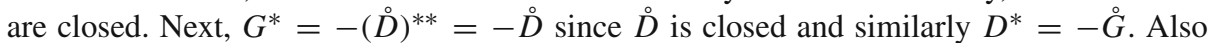
$\stackrel{\circ}{D} \subset-(\widehat{G})^{*}=-(\stackrel{\circ}{G})^{*}$, so $\stackrel{\circ}{G} \subset-(\stackrel{\circ}{D})^{*}=G$. Similarly $\stackrel{\circ}{D} \subset D$. Then, $G^{*}=-\stackrel{D}{D} \subset-D$ as required.

The classical example for this paper is as follows. Note that we do not assume any condition on the boundary of $\Omega$.

Example 2.3 Let $\Omega \subset \mathbb{R}^{d}$ be open. Define $\widehat{G}: C_{c}^{\infty}(\Omega) \rightarrow L_{2}(\Omega)^{d}$ and $\widehat{D}: C_{c}^{\infty}(\Omega)^{d} \rightarrow$ $L_{2}(\Omega)$ by

$$
\widehat{G} u=\left(\partial_{1} u, \ldots, \partial_{d} u\right) \text { and } \widehat{D} q=\sum_{k=1}^{d} \partial_{k} q_{k} .
$$

Define $H_{0}=L_{2}(\Omega)$ and $H_{1}=L_{2}(\Omega)^{d}$. Then, (2) in Example 2.2 follows from integration by parts. The associated operators are denoted by $G=\operatorname{grad}, \stackrel{\circ}{G}=\operatorname{grad}, D=\operatorname{div}$ and $\dot{D}=$ div. It is not hard to show that $\operatorname{dom}(\operatorname{grad})=H_{0}^{1}(\Omega)$, dom $(\operatorname{grad})=H^{1}(\Omega)$ and $\operatorname{dom}(\operatorname{div})=H_{\operatorname{div}}(\Omega)=\left\{q \in L_{2}(\Omega)^{d}: \operatorname{div} q \in L_{2}(\Omega)\right\}$.

We next define an (abstract) variant of the trace spaces $H^{1 / 2}(\Gamma)$ and $H^{-1 / 2}(\Gamma)$. Throughout this paper, we provide the domain of an operator with the graph norm. Define

$$
\mathrm{BD}(G)=\operatorname{dom}(\stackrel{\circ}{G})^{\perp_{\operatorname{dom}(G)}} \quad \text { and } \quad \mathrm{BD}(D)=\operatorname{dom}(\stackrel{D}{D})^{\perp_{\operatorname{dom}(D)}} .
$$

We provide $\operatorname{BD}(G)$ and $\operatorname{BD}(D)$ with the induced inner products of $\operatorname{dom}(G)$ and $\operatorname{dom}(D)$. We denote by $\pi_{\mathrm{BD}(G)}$ and $\pi_{\mathrm{BD}(D)}$ the corresponding projections onto $\mathrm{BD}(G)$ and $\operatorname{BD}(D)$, respectively.

Example 2.4 Let $\Omega, G$ and $D$ be as in Example 2.3. Then, $\operatorname{BD}(G)=\left\{u \in H^{1}(\Omega): \Delta u=\right.$ $u$ weakly on $\Omega$ \}. Indeed, let $u \in \operatorname{BD}(G)$. Then, $u \in H^{1}(\Omega)$ and $0=(u, v)_{\operatorname{dom}(G)}=$ $(u, v)_{L_{2}(\Omega)}+(\operatorname{grad} u, \operatorname{grad} v)_{L_{2}(\Omega)}$ for all $v \in \operatorname{dom}(\stackrel{\circ}{G})=H_{0}^{1}(\Omega)$. So $\Delta u=u$ weakly on $\Omega$. The converse inclusion is similar.

Lemma 2.5 $\mathrm{BD}(G)=\operatorname{ker}(I-D G)$ and $\mathrm{BD}(D)=\operatorname{ker}(I-G D)$.

Proof By Remark 2.1, it suffices to prove the first equality. Let $u \in \operatorname{BD}(G)$. Then,

$$
(u, v)_{H_{0}}+(G u, \stackrel{\circ}{G} v)_{H_{1}}=(u, v)_{\operatorname{dom}(G)}=0
$$

for all $v \in \operatorname{dom}(\stackrel{\circ}{G})$. So $G u \in \operatorname{dom}\left((\stackrel{\circ}{G})^{*}\right)=\operatorname{dom}(D)$ and $D G u=-(\stackrel{\circ}{G})^{*} G u=u$. Therefore, $u \in \operatorname{ker}(I-D G)$. The converse follows similarly. 
Corollary 2.6 If $u \in \mathrm{BD}(G)$, then $G u \in \mathrm{BD}(D)$. If $q \in \mathrm{BD}(D)$, then $D q \in \mathrm{BD}(G)$.

Proof Let $u \in \operatorname{BD}(G)$. Then, $u \in \operatorname{dom}(D G)$ and $D G u=u \in \operatorname{dom}(D G)$. Therefore, $u \in \operatorname{dom}(G D G)$ and $(I-G D) G u=G(I-D G) u=0$. So $G u \in \operatorname{ker}(I-G D)=\mathrm{BD}(D)$ by Lemma 2.5 . The other statement follows similarly.

Define $\dot{G}: \mathrm{BD}(G) \rightarrow \mathrm{BD}(D)$ and $\dot{D}: \mathrm{BD}(D) \rightarrow \mathrm{BD}(G)$ by

$$
\dot{G} u=G u \text { and } \dot{D} q=D q .
$$

Lemma 2.7 The operators $\dot{G}$ and $\dot{D}$ are unitary. Moreover, $(\dot{G})^{*}=\dot{D}$.

Proof See [13, Theorem 5.2]. For the convenience of the reader, we include the proof. Clearly, $\dot{D} \dot{G}=I_{\mathrm{BD}(G)}$ and $\dot{G} \dot{D}=I_{\mathrm{BD}(D)}$ by Lemma 2.5. Moreover,

$$
\begin{aligned}
(\dot{G} u, q)_{\mathrm{BD}(D)} & =(\dot{G} u, q)_{H_{1}}+(\dot{D} \dot{G} u, \dot{D} q)_{H_{0}}=(\dot{G} u, \dot{G} \dot{D} q)_{H_{1}}+(u, \dot{D} q)_{H_{0}} \\
& =(u, \dot{D} q)_{\mathrm{BD}(G)}
\end{aligned}
$$

for all $u \in \operatorname{BD}(G)$ and $q \in \operatorname{BD}(D)$, from which the lemma follows.

In the situation of Example 2.3, the space $\operatorname{BD}(G)$ models the boundary data of an $H^{1}(\Omega)$ function if $\Omega$ is a bounded Lipschitz domain, as shown in [16, Corollary 4.4]. Indeed, let $\Gamma=\partial \Omega$. Since $\operatorname{Tr}: H^{1}(\Omega) \rightarrow H^{1 / 2}(\Gamma)$ is continuous, surjective and $\operatorname{ker} \operatorname{Tr}=H_{0}^{1}(\Omega)=$ $\operatorname{dom}($ grad), it follows that

$$
\left.\operatorname{Tr}\right|_{\mathrm{BD}(G)}: \mathrm{BD}(G) \rightarrow H^{1 / 2}(\Gamma)
$$

is bijective and hence a topological isomorphism.

We next consider the space $\operatorname{BD}(D)$. Denote by $\operatorname{BD}(G)^{\prime}$ the space of all antilinear continuous maps from $\operatorname{BD}(G)$ into $\mathbb{C}$. There is a natural unitary map from $\operatorname{BD}(D)$ onto $\operatorname{BD}(G)^{\prime}$.

Proposition 2.8 Define $\Phi: \mathrm{BD}(D) \rightarrow \mathrm{BD}(G)^{\prime}$ by

$$
(\Phi(q))(u)=(D q, u)_{H_{0}}+(q, G u)_{H_{1}} .
$$

Then, $\Phi$ is unitary.

Proof Let $q \in \mathrm{BD}(D)$ and $u \in \mathrm{BD}(G)$. Then,

$$
\begin{aligned}
(\Phi(q))(u) & =(D q, u)_{H_{0}}+(q, G u)_{H_{1}} \\
& =(q, G u)_{H_{1}}+(D q, D G u)_{H_{0}}=(q, G u)_{\operatorname{dom}(D)}=(q, \dot{G} u)_{\mathrm{BD}(D)} .
\end{aligned}
$$

Then, the proposition follows from Lemma 2.7 and the Riesz representation theorem.

For clarity and contrast, we include the proof of the next proposition. We provide $\operatorname{Tr} H^{1}(\Omega)$ with the quotient norm.

Proposition 2.9 Let $\Omega \subset \mathbb{R}^{d}$ be open, bounded with Lipschitz boundary. Then, one has the following.

(a) For all $q \in H_{\mathrm{div}}(\Omega)$, there exists a unique $Q \in\left(\operatorname{Tr} H^{1}(\Omega)\right)^{\prime}$ such that

$$
\langle Q, \operatorname{Tr} u\rangle_{\left(\operatorname{Tr} H^{1}(\Omega)\right)^{\prime} \times \operatorname{Tr} H^{1}(\Omega)}=\int_{\Omega}(\operatorname{div} q) \bar{u}+\int_{\Omega} q \cdot \overline{\nabla u}
$$

for all $u \in H^{1}(\Omega)$. 
(b) If $q \in \operatorname{dom}($ div), then $Q=0$, where $Q$ is as in (5).

(c) If $q \in H^{1}(\Omega)^{d}$, then $Q=v \cdot \operatorname{Tr} q$, where $v$ is the outward normal vector on the boundary $\Gamma$ of $\Omega$ and $Q$ is as in (5).

Proof '(a)'. Define $F: H^{1}(\Omega) \rightarrow \mathbb{C}$ by

$$
F(u)=\int_{\Omega}(\operatorname{div} q) \bar{u}+\int_{\Omega} q \cdot \overline{\nabla u} .
$$

Then, $F \in H^{1}(\Omega)^{\prime}$. Moreover, if $u \in H_{0}^{1}(\Omega)$, then $F(u)=0$. Hence, there exists a unique continuous antilinear map $\widetilde{F}: \operatorname{Tr} H^{1}(\Omega) \rightarrow \mathbb{C}$ such that $\widetilde{F}(\operatorname{Tr} u)=F(u)$ for all $u \in H^{1}(\Omega)$. Then, the first statement follows.

'(b)'. We use the notation as in Example 2.3. Let $q \in \operatorname{dom}(\stackrel{D}{D})$. Since $\stackrel{\circ}{D}=-G^{*}$ one deduces that $F(u)=\int_{\Omega}(\operatorname{div} q) \bar{u}+\int_{\Omega} q \cdot \overline{\nabla u}=(\stackrel{D}{D} q, u)_{H_{1}}+(q, G u)_{H_{0}}=0$ for all $u \in$ $\operatorname{dom}(G)$. So $Q=0$, because $\operatorname{dom}(G)$ is dense in $H^{1}(\Omega)$.

'(c)'. Suppose that $q \in H^{1}(\Omega)^{d}$. Let $u \in H^{1}(\Omega)$. Then, $\bar{u} q \in W^{1,1}(\Omega)^{d}$ and the divergence theorem gives

$$
\int_{\Omega}(\operatorname{div} q) \bar{u}+\int_{\Omega} q \cdot \overline{\nabla u}=\int_{\Omega} \operatorname{div}(\bar{u} q)=\int_{\Gamma} v \cdot \operatorname{Tr}(\bar{u} q)=\int_{\Gamma}(v \cdot \operatorname{Tr} q) \operatorname{Tr} \bar{u} .
$$

So $Q=v \cdot \operatorname{Tr} q$.

If $q \in H_{\mathrm{div}}(\Omega)$, and $Q$ and $v$ are as in Proposition 2.9, then we define $(v q)=Q$. So $(v q)=v \cdot \operatorname{Tr} q$ if $q \in H^{1}(\Omega)^{d}$.

Example 2.10 Let $\Omega$ be a bounded Lipschitz domain with boundary $\Gamma$. Let $G$ and $D$ be as in Example 2.3. Let $\Phi$ be as in Proposition 2.8. Then,

$$
(\Phi(q))(u)=\langle(v q), \operatorname{Tr} u\rangle_{\left(\operatorname{Tr} H^{1}(\Omega)\right)^{\prime} \times \operatorname{Tr} H^{1}(\Omega)}
$$

for all $q \in \mathrm{BD}(D)$ and $u \in \mathrm{BD}(G)$.

It follows from (3) and Proposition 2.8 that the spaces $\operatorname{BD}(D)$ and $H^{-1 / 2}(\Gamma)$ are isomorphic. Hence, $\dot{G}$ is a variant of the Dirichlet-to-Neumann operator.

Next, we introduce the (variable) coefficients for our abstract Dirichlet-to-Neumann operator. Recall that a bounded operator $M$ in a Hilbert space $H$ is called coercive if there exists a $\mu>0$ such that $\operatorname{Re} M \geqslant \mu I$, where $\operatorname{Re} M=\frac{1}{2}\left(M+M^{*}\right)$. That is, $M$ is coercive if and only if there exists a $\mu>0$ such that $\operatorname{Re}(M x, x) \geqslant \mu\|x\|_{H}^{2}$ for all $x \in H$.

As for the classical Dirichlet-to-Neumann operator, we first show that the Dirichlet problem has a unique solution.

Proposition 2.11 Let $a \in \mathcal{L}\left(H_{1}\right)$ and $m \in \mathcal{L}\left(H_{0}\right)$ be coercive. Let $u_{0} \in \mathrm{BD}(G)$. Then, there exists a unique $u \in \operatorname{dom}(D a G)$ such that $m u-D a G u=0$ and $u-u_{0} \in \operatorname{dom}(\stackrel{\circ}{)})$.

For the proof of the proposition, we need several auxiliary results.

Lemma 2.12 Let $H$ be a Hilbert space, $M \in \mathcal{L}(H)$ and $A$ a skew-adjoint operator in $H$. Let $\lambda>0$ and assume that $\operatorname{Re}(M x, x)_{H} \geqslant \lambda\|x\|_{H}^{2}$ for all $x \in H$. Then, the operator $M+A$ is invertible. Moreover, the operator $(M+A)^{-1}$ is bounded from $H$ into $\operatorname{dom}(A)$ and $\left\|(M+A)^{-1}\right\|_{H \rightarrow \operatorname{dom}(A)} \leqslant \frac{1+\lambda+\|M\|}{\lambda}$. 
Proof If $x \in \operatorname{dom}(A)$, then $\operatorname{Re}((M+A) x, x)_{H}=\operatorname{Re}\left((M x, x)_{H} \geqslant \lambda\|x\|_{H}^{2}\right.$. Hence, $M+A$ is one-to-one, its range is closed, and $M+A$ is continuously invertible on its range. Since $\operatorname{Re}(M x, x)_{H}=\operatorname{Re}\left(M^{*} x, x\right)_{H}$ for all $x \in H$, we obtain similarly that $(M+A)^{*}=M^{*}-A$ is one-to-one. Therefore, $M+A$ is onto. So $M+A$ is invertible and $\left\|(M+A)^{-1}\right\|_{H \rightarrow H} \leqslant \frac{1}{\lambda}$.

Since $A(M+A)^{-1}=I-M(M+A)^{-1}$, the operator $A(M+A)^{-1}$ is bounded from $H$ into $H$ and the estimate follows.

Next, we consider matrix operators.

Lemma 2.13 Let $a \in \mathcal{L}\left(H_{1}\right)$ and $m \in \mathcal{L}\left(H_{0}\right)$ be coercive.

(a) The operators $\left(\begin{array}{cc}m & -\stackrel{\circ}{D} \\ -G & a^{-1}\end{array}\right)$ and $\left(\begin{array}{cc}m & -D \\ -\stackrel{\circ}{G} & a^{-1}\end{array}\right)$ in $H_{0} \times H_{1}$ are invertible.

(b) The operator $\left(\begin{array}{cc}m & -\stackrel{\circ}{D} \\ -G & a^{-1}\end{array}\right)^{-1}$ is bounded from $H_{0} \times H_{1}$ into $\operatorname{dom}(G) \times \operatorname{dom}(\stackrel{D}{)})$.

(c) The operator $\left(\begin{array}{cc}m & -D \\ -\stackrel{\circ}{G} & a^{-1}\end{array}\right)^{-1}$ is bounded from $H_{0} \times H_{1}$ into $\operatorname{dom}(\stackrel{\circ}{G}) \times \operatorname{dom}(D)$.

Proof Let $H=H_{0} \times H_{1}, M=\left(\begin{array}{cc}m & 0 \\ 0 & a^{-1}\end{array}\right)$ and $A=\left(\begin{array}{cc}0 & -\stackrel{\circ}{D} \\ -G & 0\end{array}\right)$ with $\operatorname{dom}(A)=\operatorname{dom}(G) \times$

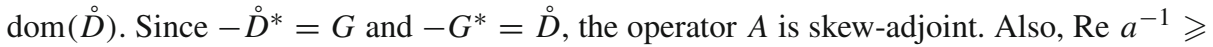
$\|a\|^{-2} \operatorname{Re} a$, so $M$ is coercive. Therefore, $M+A$ is invertible and the operator $(M+A)^{-1}$ is bounded from $H$ into $\operatorname{dom}(A)$ by Lemma 2.12. This proves the first part of Statement (a) and Statement (b)

The remaining parts of the lemma follow similarly.

Lemma 2.14 Let $a \in \mathcal{L}\left(H_{1}\right)$ and $m \in \mathcal{L}\left(H_{0}\right)$ be coercive. Let $u \in \operatorname{dom}(G), q \in \operatorname{dom}(D)$, $u_{0} \in \mathrm{BD}(G)$ and $q_{0} \in \mathrm{BD}(D)$.

(a) The following conditions are equivalent.

(i) $D q=m u, q=a G u$ and $u-u_{0} \in \operatorname{dom}(\stackrel{\circ}{G})$.

(ii) $q=a G u, u-u_{0} \in \operatorname{dom}(\stackrel{\circ}{G})$ and

$$
(a G u, \stackrel{\circ}{G} v)_{H_{1}}=-(m u, v)_{H_{0}}
$$

for all $v \in \operatorname{dom}(\stackrel{\circ}{G})$.

(iii) $\left(\begin{array}{c}u-u_{0} \\ q\end{array}\right)=\left(\begin{array}{cc}m & -D \\ -\stackrel{\circ}{G} & a^{-1}\end{array}\right)^{-1}\left(\begin{array}{c}-m u_{0} \\ G u_{0}\end{array}\right)$.

(b) The following conditions are equivalent.

(i) $D q=m u, q=a G u$ and $q-q_{0} \in \operatorname{dom}(\stackrel{D}{D})$.

(ii) $\left(\begin{array}{c}u \\ q-q_{0}\end{array}\right)=\left(\begin{array}{cc}m & -\stackrel{\circ}{D} \\ -G & a^{-1}\end{array}\right)^{-1}\left(\begin{array}{c}D q_{0} \\ -a^{-1} q_{0}\end{array}\right)$.

Proof '(a)'. '(i) $\Leftrightarrow$ (ii)'. This follows immediately from the equality $D=-(\stackrel{\circ}{G})^{*}$.

'(i) $\Leftrightarrow$ (iii)'. By a simple algebraic manipulation, Condition (i) is equivalent to

$$
u-u_{0} \in \operatorname{dom}(\stackrel{\circ}{G}) \text { and }\left(\begin{array}{cc}
m & -D \\
-G & a^{-1}
\end{array}\right)\left(\begin{array}{c}
u-u_{0} \\
q
\end{array}\right)=\left(\begin{array}{c}
-m u_{0} \\
G u_{0}
\end{array}\right) \text {. }
$$

By Lemma 2.13(a), this is equivalent to Condition (iii).

'(b)'. The proof is similar. 
Now, we are able to prove Proposition 2.11.

Proof of Proposition 2.11 First we show existence. Let $u \in \operatorname{dom}(G)$ and $q \in \operatorname{dom}(D)$ be such that

$$
\left(\begin{array}{c}
u-u_{0} \\
q
\end{array}\right)=\left(\begin{array}{cc}
m & -D \\
-\stackrel{\circ}{G} & a^{-1}
\end{array}\right)^{-1}\left(\begin{array}{c}
-m u_{0} \\
G u_{0}
\end{array}\right) .
$$

Then, $u$ satisfies the desired properties by Lemma 2.14(a) (iii) $\Rightarrow$ (i).

It remains to show uniqueness. Let $\tilde{u} \in \operatorname{dom}(D a G)$ and suppose that $m \tilde{u}-D a G \tilde{u}=0$ and $\tilde{u}-u_{0} \in \operatorname{dom}(\stackrel{\circ}{G})$. Set $\tilde{q}=a G \tilde{u}$. Then, it follows from Lemma 2.14(a) (i) $\Rightarrow$ (iii) that

$$
\left(\begin{array}{c}
\tilde{u}-u_{0} \\
\tilde{q}
\end{array}\right)=\left(\begin{array}{cc}
m & -D \\
-\stackrel{\circ}{G} & a^{-1}
\end{array}\right)^{-1}\left(\begin{array}{c}
-m u_{0} \\
G u_{0}
\end{array}\right),
$$

which implies that $u=\tilde{u}$.

There is a similar version of Proposition 2.11 for the Neumann problem.

Proposition 2.15 Let $a \in \mathcal{L}\left(H_{1}\right)$ and $m \in \mathcal{L}\left(H_{0}\right)$ be coercive. Let $q_{0} \in \mathrm{BD}(D)$. Then, there exists a unique $u \in \operatorname{dom}(D a G)$ such that $m u-D a G u=0$ and $a G u-q_{0} \in \operatorname{dom}(\stackrel{D}{D})$.

Proof This follows similarly to the proof of Proposition 2.11, but now use Lemma 2.14(b) instead of Lemma 2.14(a).

At this stage, we are able to define the Dirichlet-to-Neumann operator with variable coefficients as an operator acting from $\operatorname{BD}(G)$ (the abstract realization of $H^{1 / 2}(\Gamma)$ ) to $\operatorname{BD}(D)$ (the abstract realization of $H^{-1 / 2}(\Gamma)$ ).

Definition 2.16 Let $a \in \mathcal{L}\left(H_{1}\right)$ and $m \in \mathcal{L}\left(H_{0}\right)$ be coercive. Define the operator

$$
\Lambda: \mathrm{BD}(G) \rightarrow \mathrm{BD}(D)
$$

as follows. Let $u_{0} \in \operatorname{BD}(G)$. By Proposition 2.11, there exists a unique $u \in \operatorname{dom}(\operatorname{DaG})$ such that $m u-D a G u=0$ and $u-u_{0} \in \operatorname{dom}(\stackrel{\circ}{G})$. Then, we define $\Lambda u_{0}=\pi_{\mathrm{BD}(D)} a G u$. We call $\Lambda$ the Dirichlet-to-Neumann operator associated with $-D a G+m$.

So the graph of the operator $\Lambda$ is equal to

$$
\left\{\left(\pi_{\mathrm{BD}(G)} u, \pi_{\mathrm{BD}(D)} a G u\right): u \in \operatorname{dom}(D a G) \text { and } m u-D a G u=0\right\} .
$$

Theorem 2.17 Let $a \in \mathcal{L}\left(H_{1}\right)$ and $m \in \mathcal{L}\left(H_{0}\right)$ be coercive. Then, the operator $\Lambda$ associated with $-D a G+m$ is bounded and invertible. Moreover,

$$
\Lambda u_{0}=\left(\begin{array}{ll}
0 \pi_{\mathrm{BD}(D)}
\end{array}\right)\left(\begin{array}{cc}
m & -D \\
-\stackrel{\circ}{G} & a^{-1}
\end{array}\right)^{-1}\left(\begin{array}{c}
-m \\
G
\end{array}\right) u_{0}
$$

for all $u_{0} \in \mathrm{BD}(G)$ and

$$
\Lambda^{-1} q_{0}=\left(\begin{array}{ll}
\pi_{\mathrm{BD}(G)} & 0
\end{array}\right)\left(\begin{array}{cc}
m & -\stackrel{\circ}{D} \\
-G & a^{-1}
\end{array}\right)^{-1}\left(\begin{array}{c}
D \\
-a^{-1}
\end{array}\right) q_{0}
$$

for all $q_{0} \in \mathrm{BD}(D)$.

Proof The expression for $\Lambda$ follows from Lemma 2.14(a), arguing as in the proof of Proposition 2.11. The boundedness of $\Lambda$ is then a consequence of Lemma 2.13(c).

The proof for $\Lambda^{-1}$ is similar, using Lemma 2.14(b), Proposition 2.15 and Lemma 2.13(b). 


\section{An intermediate operator and m-sectoriality}

In Proposition 2.8, we showed that the space $\operatorname{BD}(D)$ is naturally isomorphic to $\operatorname{BD}(G)^{\prime}$. In this section, we assume that there is a Hilbert space $H$ such that $\operatorname{BD}(G) \hookrightarrow H \hookrightarrow \operatorname{BD}(G)^{\prime}$ is a Gelfand triple. Then, we study the part of the Dirichlet-to-Neumann operator in $H$. In the model example, Example 2.3, one can take $H=L_{2}(\Gamma)$.

Throughout this section, we adopt the notation and assumptions as in the beginning of Sect. 2. In addition, let $H$ be a Hilbert space and $\kappa \in \mathcal{L}(\operatorname{BD}(G), H)$. We assume that $\kappa$ is one-to-one and has dense range.

Example 3.1 Let $\Omega$ be a bounded Lipschitz domain with boundary $\Gamma$. Let $G$ and $D$ be as in Example 2.3. Let $\sigma \in\left(-\infty, \frac{1}{2}\right]$ and choose $H=H^{\sigma}(\Gamma)$. Define $\kappa: \operatorname{BD}(G) \rightarrow H$ by $\kappa(u)=\operatorname{Tr} u$. Then, $\kappa$ is one-to-one and has dense range. Note that $\kappa$ is compact if and only if $\sigma<\frac{1}{2}$.

Now, suppose that $\sigma=0$, so $H=L_{2}(\Gamma)$. Let $\psi \in L_{2}(\Gamma)$ and set $u=\kappa^{*} \psi$. Then, $u \in \mathrm{BD}(G)$, so $u \in H^{1}(\Omega)$ and $\Delta u=u$ weakly on $\Omega$ by Example 2.4. If $v \in \operatorname{BD}(G)$, then

$$
\begin{aligned}
\int_{\Gamma} \psi \overline{\operatorname{Tr} v} & =(\psi, \kappa(v))_{L_{2}(\Gamma)}=\left(\kappa^{*} \psi, v\right)_{\mathrm{BD}(G)}=(u, v)_{\mathrm{BD}(G)} \\
& =\int_{\Omega} u \bar{v}+\int_{\Omega} \nabla u \cdot \overline{\nabla v}=\int_{\Omega}(\Delta u) \bar{v}+\int_{\Omega} \nabla u \cdot \overline{\nabla v} .
\end{aligned}
$$

Alternatively, if $v \in H_{0}^{1}(\Omega)=\operatorname{dom}(\stackrel{\circ}{G})$, then

$$
\int_{\Gamma} \psi \overline{\operatorname{Tr} v}=0=\int_{\Omega}(\Delta u) \bar{v}+\int_{\Omega} \nabla u \cdot \overline{\nabla v} .
$$

So by linearity

$$
\int_{\Gamma} \psi \overline{\operatorname{Tr} v}=\int_{\Omega}(\Delta u) \bar{v}+\int_{\Omega} \nabla u \cdot \overline{\nabla v}
$$

for all $v \in H^{1}(\Omega)$. Hence, $u$ has a weak normal derivative and $\partial_{\nu} u=\psi$.

We consider the Gelfand triple

$$
\mathrm{BD}(G) \stackrel{\kappa}{\hookrightarrow} H \simeq H^{\prime} \stackrel{\kappa^{\prime}}{\hookrightarrow} \mathrm{BD}(G)^{\prime}
$$

with $H$ as pivot space. Recall that $\operatorname{BD}(G)^{\prime}$ is naturally isomorphic to $\operatorname{BD}(D)$ by Proposition 2.8. We aim to describe the part of the Dirichlet-to-Neumann operator $\Lambda$ in $H$. We describe the image of $H$ in $\mathrm{BD}(D)$ under the above maps $H \simeq H^{\prime} \stackrel{\kappa^{\prime}}{\hookrightarrow} \mathrm{BD}(G)^{\prime} \simeq \mathrm{BD}(D)$.

Lemma 3.2 Let $\Phi: \mathrm{BD}(D) \rightarrow \mathrm{BD}(G)^{\prime}$ be as in Proposition 2.8. Define $F: H \rightarrow H^{\prime}$ by $(F \varphi)(\psi)=(\varphi, \psi)_{H}$. Then, $\Phi^{-1} \circ \kappa^{\prime} \circ F=G \circ \kappa^{*}$.

Proof Let $\varphi \in H$ and write $q=\left(\Phi^{-1} \circ \kappa^{\prime} \circ F\right)(\varphi)$. Let $u \in \operatorname{BD}(G)$. Then, it follows from Lemma 2.7 and (4) that

$$
\begin{aligned}
(\dot{D} q, u)_{\mathrm{BD}(G)} & =(q, \dot{G} u)_{\mathrm{BD}(D)} \\
& =(\Phi(q))(u)=\left(\left(\kappa^{\prime} \circ F\right) \varphi\right)(u)=(\varphi, \kappa(u))_{H}=\left(\kappa^{*} \varphi, u\right)_{\mathrm{BD}(G)} .
\end{aligned}
$$

So $\dot{D} q=\kappa^{*} \varphi$ and $q=\dot{G} \dot{D} q=\dot{G} \kappa^{*} \varphi$.

Now, we are able to define the part of the Dirichlet-to-Neumann operator in $H$. 
Definition 3.3 Let $a \in \mathcal{L}\left(H_{1}\right)$ and $m \in \mathcal{L}\left(H_{0}\right)$ be coercive. Define the operator $\Lambda_{H}$ in $H$ as follows. Let $\varphi, \psi \in H$. Then, we say that $\varphi \in \operatorname{dom}\left(\Lambda_{H}\right)$ and $\Lambda_{H} \varphi=\psi$ if there exists a $u_{0} \in \mathrm{BD}(G)$ such that $\kappa\left(u_{0}\right)=\varphi$ and $\Lambda u_{0}=\left(G \circ \kappa^{*}\right)(\psi)$, where $\Lambda$ is the Dirichletto-Neumann operator associated with $-D a G+m$. We call $\Lambda_{H}$ the Dirichlet-to-Neumann operator in $H$ associated with $-D a G+m$.

Despite the abundance of choice of the space $H$, see Example 3.1, the operator $-\Lambda_{H}$ is always a semigroup generator.

Theorem 3.4 Let $a \in \mathcal{L}\left(H_{1}\right)$ and $m \in \mathcal{L}\left(H_{0}\right)$ be coercive. Then, the Dirichlet-to-Neumann operator $\Lambda_{H}$ associated with $-D a G+m$ is $m$-sectorial. In particular, if both a and $m$ are symmetric, then $\Lambda_{H}$ is self-adjoint.

The proof of this theorem is based on form methods and the next theorem.

Theorem 3.5 Let $\widetilde{H}, V$ be Hilbert spaces, and let $j \in \mathcal{L}(V, \widetilde{H})$ with dense range. Let $\mathfrak{b}: V \times$ $V \rightarrow \mathbb{C}$ be a continuous coercive sesquilinear form, that is, there exists a $\mu>0$ such that $\operatorname{Re} \mathfrak{b}(v) \geqslant \mu\|v\|_{V}^{2}$ for all $v \in V$. Define the operator $A$ in $\widetilde{H}$ as follows. Let $x, f \in \widetilde{H}$. Then, $x \in \operatorname{dom}(A)$ and $A x=f$ if there exists $a u \in V$ such that $j(u)=x$ and $\mathfrak{b}(u, v)=$ $(f, j(v))_{\tilde{H}}$ for all $v \in V$. Then, $A$ is well-defined and $m$-sectorial. If, in addition, $\mathfrak{b}$ is symmetric, then $A$ is self-adjoint.

Proof See [3, Theorem 2.1].

In the situation of Theorem 3.5, we call $A$ the operator associated with $(\mathfrak{b}, j)$.

Theorem 3.4 is an immediate consequence of Theorem 3.5 and the next proposition.

Proposition 3.6 Let $a \in \mathcal{L}\left(H_{1}\right)$ and $m \in \mathcal{L}\left(H_{0}\right)$ be coercive. Define the sesquilinear form $\mathfrak{b}: \operatorname{dom}(G) \times \operatorname{dom}(G) \rightarrow \mathbb{C}$ by

$$
\mathfrak{b}(u, v)=(a G u, G v)_{H_{1}}+(m u, v)_{H_{0}} .
$$

Then, $\mathfrak{b}$ is coercive and continuous. Further define $j: \operatorname{dom}(G) \rightarrow H$ by $j=\kappa \circ \pi_{\mathrm{BD}(G)}$. Then, the Dirichlet-to-Neumann operator $\Lambda_{H}$ associated with $-D a G+m$ is equal to the operator associated with $(\mathfrak{b}, j)$.

Proof The form $\mathfrak{b}$ is coercive since both $a$ and $m$ are coercive. Obviously, $\mathfrak{b}$ is continuous. Let $A$ be the operator associated with $(\mathfrak{b}, j)$. It remains to prove that $A=\Lambda_{H}$.

' $\Lambda_{H} \subset A$ '. Let $\varphi \in \operatorname{dom}\left(\Lambda_{H}\right)$ and set $\psi=\Lambda_{H} \varphi$. Then, there exists a $u_{0} \in \operatorname{BD}(G)$ with $\kappa\left(u_{0}\right)=\varphi$ and $\Lambda u_{0}=\left(G \circ \kappa^{*}\right) \psi$. By definition, there exists a $u \in \operatorname{dom}(\operatorname{DaG})$ such that $m u-D a G u=0, u-u_{0} \in \operatorname{dom}(\stackrel{\circ}{G})$ and $\Lambda u_{0}=\pi_{\mathrm{BD}(D)}(a G u)$. Then, $\left(G \circ \kappa^{*}\right) \psi=$ $\pi_{\mathrm{BD}(D)}(a G u)$ and $j(u)=\kappa \pi_{\mathrm{BD}(G)} u=\kappa\left(u_{0}\right)=\varphi$.

Next, if $v \in \operatorname{dom}(\stackrel{\circ}{G})$, then

$$
\begin{aligned}
\mathfrak{b}(u, v) & =(a G u, \stackrel{\circ}{ } v)_{H_{1}}+(m u, v)_{H_{0}} \\
& =-(D a G u, v)_{H_{0}}+(D a G u, v)_{H_{0}}=0=(\psi, 0)_{H}=(\psi, j(v))_{H} .
\end{aligned}
$$

If $v \in \mathrm{BD}(G)$, then Lemma 2.7 gives

$$
\begin{aligned}
(\psi, j(v))_{H} & =\left(\kappa^{*} \psi, v\right)_{\mathrm{BD}(G)}=\left(G \kappa^{*} \psi, G v\right)_{\mathrm{BD}(D)}=\left(\pi_{\mathrm{BD}(D)}(a G u), G v\right)_{\mathrm{BD}(D)} \\
& =(a G u, G v)_{\operatorname{dom}(D)}=(a G u, G v)_{H_{1}}+(D a G u, D G v)_{H_{0}} \\
& =(a G u, G v)_{H_{1}}+(m u, v)_{H_{0}}=\mathfrak{b}(u, v) .
\end{aligned}
$$


Since $\operatorname{dom}(G)=\operatorname{BD}(G) \oplus \operatorname{dom}(\stackrel{\circ}{G})$, it follows that $\mathfrak{b}(u, v)=(\psi, j(v))_{H}$ for all $v \in$ $\operatorname{dom}(G)$. So $\varphi \in \operatorname{dom}(A)$ and $A \varphi=\psi$.

' $A \subset \Lambda_{H}$ '. Let $\varphi \in \operatorname{dom}(A)$ and write $\psi=A \varphi$. Then, there exists a $u \in \operatorname{dom}(G)$ such that $j(u)=\varphi$ and

$$
(a G u, G v)_{H_{1}}+(m u, v)_{H_{0}}=\mathfrak{b}(u, v)=(\psi, j(v))_{H}
$$

for all $v \in \operatorname{dom}(G)$. If $v \in \operatorname{dom}(\stackrel{\circ}{G})$, then

$$
(a G u, \stackrel{\circ}{G} v)_{H_{1}}+(m u, v)_{H_{0}}=(\psi, j(v))_{H}=0 .
$$

So $a G u \in \operatorname{dom}\left((\stackrel{\circ}{G})^{*}\right)=\operatorname{dom}(D)$ and $D a G u=-(\stackrel{\circ}{G})^{*} a G u=m u$. Moreover,

$$
\Lambda \pi_{\mathrm{BD}(G)} u=\pi_{\mathrm{BD}(D)}(a G u)
$$

by the definition of $\Lambda$. Note that $\kappa\left(\pi_{\mathrm{BD}(G)} u\right)=j(u)=\varphi$.

Now, let $v \in \operatorname{BD}(G)$. Then, (6) gives

$$
\begin{aligned}
\left(\kappa^{*} \psi, v\right)_{\mathrm{BD}(G)} & =(\psi, \kappa(v))_{H} \\
& =(a G u, G v)_{H_{1}}+(m u, v)_{H_{0}} \\
& =(a G u, G v)_{H_{1}}+(D a G u, D G v)_{H_{0}} \\
& =(a G u, G v)_{\mathrm{dom}(D)} \\
& =\left(\pi_{\mathrm{BD}(D)}(a G u), G v\right)_{\mathrm{BD}(D)} \\
& =\left(D \pi_{\mathrm{BD}(G)}(a G u), v\right)_{\mathrm{BD}(G)},
\end{aligned}
$$

where we used Lemma 2.7 in the last step. So, $\kappa^{*} \psi=D \pi_{\mathrm{BD}(D)}(a G u)$. Hence,

$$
\left(G \circ \kappa^{*}\right)(\psi)=\pi_{\mathrm{BD}(D)}(a G u)=\Lambda \pi_{\mathrm{BD}(G)} u
$$

by Lemma 2.7 and (7). Therefore, $\varphi \in \operatorname{dom}\left(\Lambda_{H}\right)$ and $\Lambda_{H} \varphi=\psi$.

We next show that the operator $\Lambda_{H}$ is invertible and determine its inverse.

Proposition 3.7 The operator $\Lambda_{H}$ is invertible and

$$
\Lambda_{H}^{-1} \psi=\kappa\left(\pi_{\mathrm{BD}(G)} 0\right)\left(\begin{array}{cc}
m & -\stackrel{\circ}{D} \\
-G & a^{-1}
\end{array}\right)^{-1}\left(\begin{array}{c}
1 \\
-a^{-1} G
\end{array}\right) \kappa^{*} \psi
$$

for all $\psi \in H$.

Proof Since the form $\mathfrak{b}$ in Proposition 3.6 is coercive, it follows that the operator $\Lambda_{H}$ is invertible. Let $\varphi \in \operatorname{dom}\left(\Lambda_{H}\right)$ and write $\psi=\Lambda_{H} \varphi$. Then, there exists a $u_{0} \in \operatorname{BD}(G)$ such that $\kappa\left(u_{0}\right)=\varphi$ and $\Lambda u_{0}=G \kappa^{*} \psi$. By Theorem 2.17, we obtain that

$$
\begin{aligned}
u_{0}=\Lambda^{-1} G \kappa^{*} \psi & =\left(\pi_{\mathrm{BD}(G)} 0\right)\left(\begin{array}{cc}
m & -\stackrel{\circ}{D} \\
-G & a^{-1}
\end{array}\right)^{-1}\left(\begin{array}{c}
D \\
-a^{-1}
\end{array}\right) G \kappa^{*} \psi \\
& =\left(\begin{array}{ll}
\pi_{\mathrm{BD}(G)} & 0
\end{array}\right)\left(\begin{array}{cc}
m & -\stackrel{\circ}{D} \\
-G & a^{-1}
\end{array}\right)^{-1}\left(\begin{array}{c}
I \\
-a^{-1} G
\end{array}\right) \kappa^{*} \psi,
\end{aligned}
$$

where we used Lemma 2.7 in the last step. Next, apply $\kappa$ to both sides. Since the inverse matrix maps $H_{0} \times H_{1}$ into $\operatorname{dom}(G) \times \operatorname{dom}(D)$ by Lemma 2.13(b), the proposition follows. 


\section{Resolvent convergence}

In this section, we consider a sequence of Dirichlet-to-Neumann operators and show resolvent convergence.

Throughout this section, we adopt the notation and assumptions as in the beginning of Sect. 2. Let $H$ be a Hilbert space and $\kappa \in \mathcal{L}(\operatorname{BD}(G), H)$ injective with dense range. Further, we let $m_{n}, m \in \mathcal{L}\left(H_{0}\right)$ and $a_{n}, a \in \mathcal{L}\left(H_{1}\right)$ for all $n \in \mathbb{N}$. Let $\mu>0$ and assume that $\operatorname{Re} m_{n}, \operatorname{Re} m \geqslant \mu I_{H_{0}}$ and $\operatorname{Re} a_{n}, \operatorname{Re} a \geqslant \mu I_{H_{1}}$ for all $n \in \mathbb{N}$. Moreover, assume that $\sup _{n}\left\|a_{n}\right\|_{\mathcal{L}\left(H_{1}\right)}<\infty$. Let $\Lambda, \Lambda_{1}, \Lambda_{2}, \ldots$ be the Dirichlet-to-Neumann operators from $\mathrm{BD}(G)$ into $\mathrm{BD}(D)$ associated with $-D a G+m,-D a_{1} G+m_{1},-D a_{2} G+m_{2}, \ldots$ as in Definition 2.16. Similarly, let $\Lambda_{H}, \Lambda_{H}^{(1)}, \Lambda_{H}^{(2)}, \ldots$ be the Dirichlet-to-Neumann operators in $H$ as in Definition 3.3.

Throughout this section, we suppose in addition that the inclusion $\operatorname{dom}(G) \hookrightarrow H_{0}$ is compact.

The compactness assumption is valid in our model case, Example 2.3, if $\Omega$ is bounded and has a continuous boundary or, equivalently, if $\Omega$ has the segment property.

We state two well-known consequences of the compactness assumption.

Lemma 4.1 (a) There exists a $c>0$ such that $\|u\|_{H_{0}} \leqslant c\|G u\|_{H_{1}}$ for all $u \in \operatorname{dom}(G) \cap$ $\operatorname{ker}(G)^{\perp} H_{0}$.

(b) The space $\operatorname{ran}(G)$ is closed in $H_{1}$.

Proof '(a)'. Suppose not. Then, there exists a sequence $\left(u_{n}\right)_{n \in \mathbb{N}}$ in $\operatorname{dom}(G) \cap \operatorname{ker}(G)^{\perp} H_{0}$ such that $\left\|u_{n}\right\|_{H_{0}}=1$ and

$$
\left\|u_{n}\right\|_{H_{0}} \geqslant n\left\|G u_{n}\right\|_{H_{1}}
$$

for all $n \in \mathbb{N}$. Then, $\left(u_{n}\right)_{n \in \mathbb{N}}$ is bounded in $\operatorname{dom}(G)$. We may assume without loss of generality that there exists a $u \in \operatorname{dom}(G)$ such that $\lim u_{n}=u$ weakly in $\operatorname{dom}(G)$. Since the inclusion $\operatorname{dom}(G) \subset H_{0}$ is compact, we obtain that $\lim u_{n}=u$ in $H_{0}$. Then, $u \in$ $\operatorname{ker}(G)^{\perp H_{0}}$ since $\operatorname{ker}(G)^{\perp} H_{0}$ is closed in $H_{0}$. Moreover, $\|u\|_{H_{0}}=1$ and in particular $u \neq 0$. Alternatively, (8) implies that $\|G u\|_{H_{1}} \leqslant \liminf _{n \rightarrow \infty}\left\|G u_{n}\right\|_{H_{1}}=0$. So $u \in \operatorname{ker}(G)$. Hence, $u \in \operatorname{ker}(G) \cap \operatorname{ker}(G)^{\perp} H_{0}=\{0\}$ and $u=0$. This is a contradiction.

'(b)'. This is a consequence of Statement (a) and the closedness of $G$.

We provide $\operatorname{ran}(G)$ with the induced norm of $H_{1}$. Throughout the remainder of this section, we denote by $\iota: \operatorname{ran}(G) \hookrightarrow H_{1}$ the embedding map. Note that $\iota^{*}$ is the orthogonal projection from $H_{1}$ onto $\operatorname{ran}(G)$. The main result of this section is the following theorem.

Theorem 4.2 Suppose that $\lim m_{n}=m$ in the weak operator topology on $\mathcal{L}\left(H_{0}\right)$ and $\lim _{n \rightarrow \infty}\left(\iota^{*} a_{n} \iota\right)^{-1}=\left(\iota^{*} a \iota\right)^{-1}$ in the weak operator topology on $\mathcal{L}(\operatorname{ran}(G))$. Then,

$$
\lim _{n \rightarrow \infty}\left(\Lambda_{H}^{(n)}\right)^{-1}=\Lambda_{H}^{-1}
$$

in the weak operator topology on $\mathcal{L}(H)$. Moreover, if in addition the map $\kappa$ is compact, then the convergence is uniform in $\mathcal{L}(H)$.

If the $m_{n}$ are multiplication operators, then convergence in the weak operator topology can be rephrased.

Example 4.3 If $\Omega \subset \mathbb{R}^{d}$ is open, $H_{0}=L_{2}(\Omega), V_{n}, V \in L_{\infty}(\Omega)$ and $m_{n}, m$ are the multiplication operators associated with $V_{n}$ and $V$ for all $n \in \mathbb{N}$, then $\lim m_{n}=m$ in the weak operator topology on $\mathcal{L}\left(H_{0}\right)$ if and only if $\lim V_{n}=V$ in the weak*-topology on $L_{\infty}(\Omega)$. 
For the proof of Theorem 4.2, we need some preliminary results. The first one contains an identity for $\Lambda$ involving $\operatorname{ran}(G)$.

Lemma 4.4 (a) Let $q \in H_{1}$. Then, $q \in \operatorname{dom}\left(\stackrel{D}{)}\right.$ if and only if $\iota^{*} q \in \operatorname{dom}(\stackrel{D}{)})$. In that case $\stackrel{\circ}{D} q=\stackrel{\circ}{D} \iota^{*} q$.

(b) The operator $\stackrel{\circ}{D} \iota^{\prime}: \operatorname{ran}(G) \cap \operatorname{dom}(\stackrel{\circ}{D}) \rightarrow H_{0}$ is a closed and densely defined operator in $\operatorname{ran}(G)$. Moreover, $(\stackrel{D}{\iota})^{*}=-\iota^{*} G$.

(c) The operator $D^{\circ} \iota$ is injective.

(d) The inclusion $\operatorname{dom}(\stackrel{D}{\mathrm{D}}) \subset H_{1}$ is compact.

(e) The operator $\left(\begin{array}{cc}m & -\stackrel{\circ}{D} \iota \\ -\iota^{*} G\left(\iota^{*} a \iota\right)^{-1}\end{array}\right): \operatorname{dom}(G) \times(\operatorname{ran}(G) \cap \operatorname{dom}(\stackrel{\circ}{D})) \rightarrow H_{0} \times \operatorname{ran}(G)$ is invertible.

(f) The operator $\left(\begin{array}{cc}m & -\stackrel{\circ}{D} \iota \\ -\iota^{*} G\left(\iota^{*} a \iota\right)^{-1}\end{array}\right)^{-1}$ is bounded from $H_{0} \times \operatorname{ran}(G)$ into $\operatorname{dom}(G) \times \operatorname{dom}(\stackrel{D}{D})$.

(g) If $q_{0} \in \mathrm{BD}(D)$, then

$$
\Lambda^{-1} q_{0}=\left(\pi_{\mathrm{BD}(G)} 0\right)\left(\begin{array}{cc}
m & -\stackrel{\circ}{D} \iota \\
-\iota^{*} G & \left(\iota^{*} a \iota\right)^{-1}
\end{array}\right)^{-1}\left(\begin{array}{c}
D \\
-\left(\iota^{*} a \iota\right)^{-1} \iota^{*}
\end{array}\right) q_{0} .
$$

Proof '(a)'. First $q-\iota^{*} q \in(\operatorname{ran}(G))^{\perp_{H_{1}}}=\operatorname{ker}\left(G^{*}\right)=\operatorname{ker}(\stackrel{D}{D}) \subset \operatorname{dom}(\stackrel{D}{D})$. This shows the equivalence. Since $\stackrel{D}{D}\left(q-\iota^{*} q\right)=0$, the last statement follows.

'(b)'. Let $q \in \operatorname{ran}(G)$. Since $\operatorname{dom}\left(D^{D}\right)$ is dense in $H_{1}$ there exists a sequence $\left(q_{n}\right)_{n \in \mathbb{N}}$ in $\operatorname{dom}(\stackrel{\circ}{D})$ such that $\lim q_{n}=q$ in $H_{1}$. Then, $\iota^{*} q_{n} \in \operatorname{ran}(G) \cap \operatorname{dom}(\stackrel{\circ}{D})$ for all $n \in \mathbb{N}$ by Statement (a) and $\lim \iota^{*} q_{n}=\iota^{*} q=q$ in $H_{1}$. So $\operatorname{ran}(G) \cap \operatorname{dom}(\stackrel{\circ}{D})$ is dense in $\operatorname{ran}(G)$.

Because $\operatorname{ran}(G)$ is closed in $H_{1}$ and $D^{\circ}$ is a closed operator, one deduces easily that the operator $\stackrel{\circ}{\llcorner}$ is closed. It remains to show that $(\stackrel{D}{\iota})^{*}=-\iota^{*} G$.

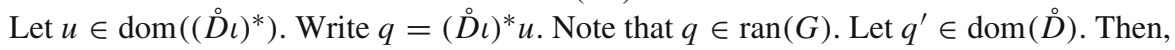
Statement (a) implies that

$$
\begin{aligned}
\left(u, \stackrel{\circ}{D} q^{\prime}\right)_{H_{0}} & =\left(u, \stackrel{\circ}{D} \iota^{*} q^{\prime}\right)_{H_{0}}=\left(u,(\stackrel{\circ}{D} \iota) \iota^{*} q^{\prime}\right)_{H_{0}} \\
& =\left((\stackrel{\circ}{D} \iota)^{*} u, \iota^{*} q^{\prime}\right)_{\operatorname{ran}(G)}=\left(q, \iota^{*} q^{\prime}\right)_{\operatorname{ran}(G)}=\left(q, q^{\prime}\right)_{H_{1}} .
\end{aligned}
$$

So $u \in \operatorname{dom}\left((D)^{*}\right)=\operatorname{dom}(G)$ and $G u=-\left(D^{*}\right)^{*} u=-q$. Therefore, $-\iota^{*} G u=q=$

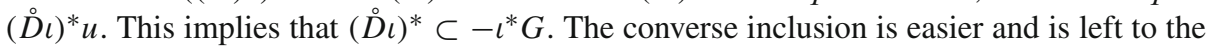
reader.

'(c)'. Let $q \in \operatorname{ran}(G) \cap \operatorname{dom}(\stackrel{D}{D})$ and suppose that $\stackrel{\circ}{D} \iota q=0$. There exists a $u \in \operatorname{dom}(G) \cap$ $(\operatorname{ker} G)^{\perp_{H_{0}}}$ such that $q=G u$. Then, $\|G u\|_{H_{1}}^{2}=-\left(q,(\stackrel{D}{D})^{*} u\right)_{H_{1}}=-(\stackrel{\circ}{D} \iota q, u)_{H_{0}}=0$. So $u \in \operatorname{ker} G$ and $u=0$.

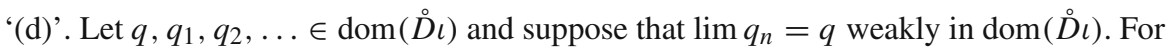
all $n \in \mathbb{N}$, there exists a unique $u_{n} \in \operatorname{dom}(G) \cap \operatorname{ker}(G)^{\perp} H_{0}$ such that $q_{n}=G u_{n}$. Since $\lim q_{n}=q$ weakly in $H_{1}$, the sequence $\left(q_{n}\right)_{n \in \mathbb{N}}$ is bounded in $H_{1}$. Hence, the sequence $\left(u_{n}\right)_{n \in \mathbb{N}}$ is bounded in $H_{0}$ by Lemma 4.1(a). Passing to a subsequence if necessary, there exists a $u \in H_{0}$ such that $\lim u_{n}=u$ weakly in $H_{0}$. Since $G$ is a weakly closed operator, one deduces that $u \in \operatorname{dom}(G)$ and $G u=q$. Then, $\lim u_{n}=u$ weakly in $\operatorname{dom}(G)$, so $\lim u_{n}=u$ strongly in $H_{0}$ by the compactness assumption. Note that $G^{*}=-\stackrel{\circ}{D}$. So

$$
\begin{aligned}
\lim _{n \rightarrow \infty}\left\|q_{n}\right\|_{H_{1}}^{2} & =\lim _{n \rightarrow \infty}\left(q_{n}, G u_{n}\right)_{H_{1}}=\lim _{n \rightarrow \infty}\left(-\stackrel{\circ}{D} q_{n}, u_{n}\right)_{H_{0}} \\
& =(-\stackrel{\circ}{D} q, u)_{H_{0}}=(q, G u)_{H_{0}}=\|q\|_{H_{1}}^{2} .
\end{aligned}
$$


Hence, $\lim q_{n}=q$ in $H_{1}$.

'(e)' and '(f)'. This is as in the proof of Lemma 2.13(a) and (b).

'(g)'. Let $q_{0} \in \mathrm{BD}(D)$. By Proposition 2.15, there exists a unique $u \in \operatorname{dom}(D a G)$ such that $m u-D a G u=0$ and $a G u-q_{0} \in \operatorname{dom}(\stackrel{D}{D})$. Then, $\Lambda^{-1} q_{0}=\pi_{B D(G)} u$. Write $q=a G u$. Then, $q-q_{0} \in \operatorname{dom}(\stackrel{\circ}{D})$, so $\stackrel{\circ}{D}\left(q-q_{0}\right)=\stackrel{\circ}{D} \iota^{*}\left(q-q_{0}\right)=(\stackrel{\circ}{D} \iota) \iota^{*}\left(q-q_{0}\right)$ by Statement (a). Therefore,

$$
D q_{0}=m u-\stackrel{\circ}{D}\left(q-q_{0}\right)=m u-\left(\stackrel{\circ}{D} \iota \iota^{*}\left(q-q_{0}\right) .\right.
$$

Also, $\iota^{*} q=\iota^{*} a G u=\left(\iota^{*} a \iota\right) \iota^{*} G u$. Hence one deduces that $\left(\iota^{*} a \iota\right)^{-1} \iota^{*} q=\iota^{*} G u$ and $-\iota^{*} G u+\left(\iota^{*} a \iota\right)^{-1} \iota^{*}\left(q-q_{0}\right)=-\left(\iota^{*} a \iota\right)^{-1} \iota^{*} q_{0}$. Together with (9), this gives

$$
\left(\begin{array}{cc}
m & -\stackrel{\circ}{D} \iota \\
-\iota^{*} G & \left(\iota^{*} a \iota\right)^{-1}
\end{array}\right)\left(\begin{array}{c}
u \\
\iota^{*}\left(q-q_{0}\right)
\end{array}\right)=\left(\begin{array}{c}
D \\
-\left(\iota^{*} a \iota\right)^{-1} \iota^{*}
\end{array}\right) q_{0}
$$

Finally, use Statement (e).

Next, we need a sequential version of Lemma 2.12.

Lemma 4.5 Let $\widetilde{H}$ be a Hilbert space, $M \in \mathcal{L}(\widetilde{H})$ and $A$ a skew-adjoint operator in $\widetilde{H}$. Further, let $\left(M_{n}\right)_{n \in \mathbb{N}}$ be a sequence in $\mathcal{L}(\widetilde{H})$ and suppose that $\lim M_{n}=M$ in the weak operator topology on $\mathcal{L}(\widetilde{H})$. Assume that the inclusion $\operatorname{dom}(A) \subset \widetilde{H}$ is compact and that there exists a $\lambda>0$ such that $\operatorname{Re} M_{n} \geqslant \lambda I_{\widetilde{H}}$ for all $n \in \mathbb{N}$. Let $\left(x_{n}\right)_{n \in \mathbb{N}}$ be a sequence in $\widetilde{H}$ which converges weakly to $x \in \widetilde{H}$. Then, $M+A$ is invertible and $\lim _{n \rightarrow \infty}\left(M_{n}+A\right)^{-1} x_{n}=$ $(M+A)^{-1} x$ weakly in $\operatorname{dom}(A)$.

Proof Obviously, $\operatorname{Re} M \geqslant \lambda I_{\widetilde{H}}$, so $M+A$ is invertible by Lemma 2.12. Consider $z_{n}=$ $\left(M_{n}+A\right)^{-1} x_{n}$ for all $n \in \mathbb{N}$. Then, $\left\|z_{n}\right\|_{\operatorname{dom}(A)} \leqslant \frac{1+\lambda+\left\|M_{n}\right\|}{\lambda}\left\|x_{n}\right\|_{\widetilde{H}}$ for all $n \in \mathbb{N}$ by Lemma 2.12. So the sequence $\left(z_{n}\right)_{n \in \mathbb{N}}$ is bounded in $\operatorname{dom}(A)$. Passing to a subsequence, we may assume without loss of generality that there exists a $z \in \operatorname{dom}(A)$ such that $\lim z_{n}=z$ weakly in $\operatorname{dom}(A)$. Then, $\lim {\underset{\sim}{n}}_{n}=z$ in $\widetilde{H}$ by the compactness assumption. Consequently, $\lim M_{n} z_{n}=M z$ weakly in $\widetilde{H}$. Now, $M_{n} z_{n}+A z_{n}=x_{n}$ for all $n \in \mathbb{N}$. Take the limit $n \rightarrow \infty$ and notice that both sides converge weakly in $\widetilde{H}$. It follows that $M z+A z=x$, so $z=(M+A)^{-1} x$. Now, the lemma follows by a standard subsequence argument.

We need one more convergence result for the proof of Theorem 4.2. This result is also of independent interest.

Proposition 4.6 Suppose that $\lim m_{n}=m$ in the weak operator topology on $\mathcal{L}\left(H_{0}\right)$ and $\lim \left(\iota^{*} a_{n} \iota\right)^{-1}=\left(\iota^{*} a \iota\right)^{-1}$ in the weak operator topology on $\mathcal{L}(\operatorname{ran}(G))$. Let $q, q_{1}, q_{2}, \ldots \in$ $\mathrm{BD}(D)$ and assume that $\lim q_{n}=q$ in $\mathrm{BD}(D)$. Then,

$$
\lim _{n \rightarrow \infty} \Lambda_{n}^{-1} q_{n}=\Lambda^{-1} q
$$

weakly in $\mathrm{BD}(G)$.

Proof Choose $\widetilde{H}=H_{0} \times \operatorname{ran}(G)$ and let $A=\left(\begin{array}{cc}0 & -\stackrel{\circ}{D} \iota \\ -\iota^{*} G & 0\end{array}\right)$ with $\operatorname{dom}(A)=\operatorname{dom}(G) \times$ $(\operatorname{ran}(G) \cap \operatorname{dom}(\stackrel{D}{D}))$. Then, $A$ is skew-adjoint in $\widetilde{H}$ by Lemma 4.4(b). Moreover, the inclusion $\operatorname{dom}(A) \subset \widetilde{H}$ is compact by Lemma 4.4(d) and the compactness assumption. Further let

$$
M=\left(\begin{array}{cc}
m & 0 \\
0 & \left(\iota^{*} a \iota\right)^{-1}
\end{array}\right) \text { and } M_{n}=\left(\begin{array}{cc}
m_{n} & 0 \\
0 & \left(\iota^{*} a_{n} \iota\right)^{-1}
\end{array}\right)
$$


for all $n \in \mathbb{N}$. Then, $\lim M_{n}=M$ in the weak operator topology on $\mathcal{L}(\widetilde{H})$. Since

$$
\operatorname{Re}\left(\iota^{*} a_{n} \iota\right)^{-1} \geqslant\left\|\iota^{*} a_{n} \iota\right\|_{\mathcal{L}(\operatorname{ran}(G))}^{-2} \operatorname{Re}\left(\iota^{*} a_{n} \iota\right) \geqslant\left\|a_{n}\right\|_{\mathcal{L}\left(H_{1}\right)}^{-2} \operatorname{Re}\left(\iota^{*} a_{n} \iota\right)
$$

for all $n \in \mathbb{N}$ and $\sup _{n}\left\|a_{n}\right\|_{\mathcal{L}\left(H_{1}\right)}<\infty$, it follows that there exists a $\lambda>0$ such that $\operatorname{Re} M_{n} \geqslant \lambda I$ for all $n \in \mathbb{N}$. We use Lemma $4.4(\mathrm{~g})$ for $\Lambda^{-1}$ and $\Lambda_{n}^{-1}$. Obviously,

$$
\lim _{n \rightarrow \infty}\left(D q_{n},-\left(\iota^{*} a_{n} \iota\right)^{-1} \iota^{*} q_{n}\right)=\left(D q,-\left(\iota^{*} a \iota\right)^{-1} \iota^{*} q\right)
$$

weakly in $\widetilde{H}$. Hence,

$$
\lim _{n \rightarrow \infty}\left(\begin{array}{cc}
m_{n} & -\stackrel{\circ}{D} \iota \\
-\iota^{*} G\left(\iota^{*} a_{n} \iota\right)^{-1}
\end{array}\right)^{-1}\left(\begin{array}{c}
D \\
-\left(\iota^{*} a_{n} \iota\right)^{-1} \iota^{*}
\end{array}\right) q_{n}=\left(\begin{array}{cc}
m & -\stackrel{\circ}{D} \iota \\
-\iota^{*} G\left(\iota^{*} a \iota\right)^{-1}
\end{array}\right)^{-1}\left(\begin{array}{c}
D \\
-\left(\iota^{*} a \iota\right)^{-1} \iota^{*}
\end{array}\right) q
$$

weakly in $\operatorname{dom}(A)$ by Lemma 4.5 . Consequently, $\lim \Lambda_{n}^{-1} q_{n}=\Lambda^{-1} q$ weakly in $\operatorname{BD}(G)$ by Lemma 4.4(g).

Now, we are able to prove the main theorem of this section.

Proof of Theorem 4.2 Let $\psi \in H$. Then, $\lim \Lambda_{n}^{-1} G \kappa^{*} \psi=\Lambda^{-1} G \kappa^{*} \psi$ weakly in $\operatorname{BD}(G)$ by Proposition 4.6. Hence,

$$
\lim _{n \rightarrow \infty}\left(\Lambda_{H}^{(n)}\right)^{-1} \psi=\lim _{n \rightarrow \infty} \kappa \Lambda_{n}^{-1} G \kappa^{*} \psi=\kappa \Lambda^{-1} G \kappa^{*} \psi=\Lambda_{H}^{-1} \psi
$$

weakly in $H$. This proves the first statement in Theorem 4.2.

Now, suppose that $\kappa$ is compact. Suppose $\lim \left(\Lambda_{H}^{(n)}\right)^{-1}=\Lambda_{H}^{-1}$ in $\mathcal{L}(H)$ is false. Passing to a subsequence if necessary, there exist $\delta>0$ and $\psi_{1}, \psi_{2}, \ldots \in H$ such that

$$
\left\|\left(\Lambda_{H}^{(n)}\right)^{-1} \psi_{n}-\Lambda_{H}^{-1} \psi_{n}\right\|_{H}>\delta\left\|\psi_{n}\right\|_{H}
$$

for all $n \in \mathbb{N}$. Without loss of generality, we may assume that $\left\|\psi_{n}\right\|_{H}=1$ for all $n \in \mathbb{N}$. Passing again to a subsequence if necessary, there exists a $\psi \in H$ such that $\lim \psi_{n}=\psi$ weakly in $H$. Then, $\lim \kappa^{*} \psi_{n}=\kappa^{*} \psi$ in $\operatorname{BD}(G)$ since $\kappa$ is compact. Therefore, $\lim G \kappa^{*} \psi_{n}=G \kappa^{*} \psi$ in $\operatorname{BD}(D)$. Hence, $\lim \Lambda_{n}^{-1} G \kappa^{*} \psi_{n}=\Lambda^{-1} G \kappa^{*} \psi$ weakly in $\operatorname{BD}(G)$ by Proposition 4.6. Using again that $\kappa$ is compact, it follows that $\lim \left(\Lambda_{H}^{(n)}\right)^{-1} \psi_{n}=\left(\Lambda_{H}\right)^{-1} \psi$ in $H$. Similarly, $\lim \left(\Lambda_{H}\right)^{-1} \psi_{n}=\left(\Lambda_{H}\right)^{-1} \psi$ in $H$. So $\lim \left\|\left(\Lambda_{H}^{(n)}\right)^{-1} \psi_{n}-\Lambda_{H}^{-1} \psi_{n}\right\|_{H}=0$. This contradicts (10) for large $n$.

In Example 6.1 and Proposition 6.2, we show that in the setting of the classical example (Example 2.3) $H$-convergence implies $\lim _{n \rightarrow \infty}\left(\iota^{*} a_{n} \iota\right)^{-1}=\left(\iota^{*} a \iota\right)^{-1}$ in the weak operator topology of $\mathcal{L}(\operatorname{ran}(G))$. Moreover, we compare it with a condition introduced in Sect. 6. In the real symmetric case, we prove in Example 6.7 that all are actually equivalent.

\section{The non-coercive case}

In this section, we drop the coerciveness condition on $m$. As a result, the Dirichlet-toNeumann operator can become multi-valued, that is, it is a graph and no longer an operator. The Dirichlet-to-Neumann graph associated with the Schrödinger operator $-\Delta+m$ has been studied in [4] and [6].

Throughout this section, we adopt the notation and assumptions as in the beginning of Sect. 2. Further, we fix an element $m \in \mathcal{L}\left(H_{0}\right)$ and a coercive $a \in \mathcal{L}\left(H_{1}\right)$. We emphasize that we do not require that $m$ is coercive. The definition of the Dirichlet-to-Neumann graph, however, remains the same as in the single-valued case in Definition 2.16. 


\section{Definition 5.1 Set}

$\Lambda=\left\{\left(\pi_{\mathrm{BD}(G)} u, \pi_{\mathrm{BD}(D)} a G u\right) \in \mathrm{BD}(G) \times \mathrm{BD}(D): u \in \operatorname{dom}(D a G)\right.$ and $\left.m u-D a G u=0\right\}$.

We call $\Lambda$ the Dirichlet-to-Neumann graph associated with $-D a G+m$.

We briefly recall some definitions in the area of (linear) graphs. Let $H, K$ be Hilbert spaces. Then, a graph $A$ is a vector subspace of $H \times K$. The domain, multi-valued part and inverse of $A$ are defined by

$$
\begin{aligned}
\operatorname{dom}(A) & =\{h \in H: \text { there exists a } k \in K \text { such that }(h, k) \in A\}, \\
\operatorname{mul}(A) & =\{k \in K:(0, k) \in A\} \text { and } \\
A^{-1} & =\{(k, h) \in K \times H:(h, k) \in A\} .
\end{aligned}
$$

We say that $A$ is single-valued or an operator if $\operatorname{mul}(A)=\{0\}$. The next lemma is trivial.

Lemma 5.2 (a) $\operatorname{mul}(\Lambda)=\left\{\pi_{\mathrm{BD}(D)} a G u: u \in \operatorname{ker}(m-D a \stackrel{\circ}{)})\right\}$.

(b) If $\operatorname{ker}(m-D a \stackrel{G}{)})=\{0\}$, then $\Lambda$ is single-valued.

As in Proposition 3.6, define the sesquilinear form $\mathfrak{b}: \operatorname{dom}(G) \times \operatorname{dom}(G) \rightarrow \mathbb{C}$ by

$$
\mathfrak{b}(u, v)=(a G u, G v)_{H_{1}}+(m u, v)_{H_{0}} .
$$

We also need the Dirichlet version of $\mathfrak{b}$ defined by $\stackrel{\mathfrak{b}}{=}=\left.\mathfrak{b}\right|_{\operatorname{dom}(\stackrel{\circ}{G}) \times \operatorname{dom}(\stackrel{\circ}{G})}$. Then, $\mathfrak{b}$ and $\mathfrak{b}$ are continuous. Hence, there exist $T \in \mathcal{L}(\operatorname{dom}(G))$ and $\stackrel{\circ}{T} \in \mathcal{L}(\operatorname{dom}(\stackrel{\circ}{G}))$ such that $\mathfrak{b}(u, v)=$ $(T u, v)_{\operatorname{dom}(G)}$ for all $u, v \in \operatorname{dom}(G)$ and $\stackrel{b}{b}(u, v)=(\stackrel{\circ}{T} u, v)_{\operatorname{dom}(\stackrel{\circ}{G})}$ for all $u, v \in \operatorname{dom}(\stackrel{\circ}{)})$. Note that $\operatorname{ker}(\stackrel{\circ}{T})=\operatorname{ker}(m-D a \stackrel{\circ}{G})$, since $(\stackrel{\circ}{G})^{*}=-D$.

With a condition on $\operatorname{ran}(\stackrel{\circ}{T})$, we can characterize the domain of the Dirichlet-to-Neumann graph $\Lambda$.

Proposition 5.3 Suppose that $\operatorname{ran}(\stackrel{\circ}{T})$ is closed in $\operatorname{dom}(\stackrel{\circ}{G})$. Then, $\operatorname{dom}(\Lambda)=\left\{u_{0} \in \mathrm{BD}(G):\left(G u_{0}, \pi_{\mathrm{BD}(D)} a^{*} G v\right)_{\mathrm{BD}(D)}=0\right.$ for all $\left.v \in \operatorname{ker}\left(m^{*}-D a^{*} \stackrel{\circ}{G}\right)\right\}$.

Proof ' $\subset$ '. Let $u_{0} \in \operatorname{dom}(\Lambda)$. Then, there exists a $u \in \operatorname{dom}(G)$ such that $m u-D a G u=0$ and $u_{0}=\pi_{B D(G)} u$. Let $v \in \operatorname{dom}(\stackrel{\circ}{G})$. Then, $(m u, v)_{H_{0}}=(D a G u, v)_{H_{0}}=-(a G u, \stackrel{\circ}{G} v)_{H_{1}}$ and

$$
\begin{aligned}
\left(\stackrel{\circ}{T}\left(u-u_{0}\right), v\right)_{\operatorname{dom}(\stackrel{\circ}{G})}=\stackrel{\circ}{\mathfrak{b}}\left(u-u_{0}, v\right) & =\left(a G\left(u-u_{0}\right), \stackrel{\circ}{G} v\right)_{H_{1}}+\left(m\left(u-u_{0}\right), v\right)_{H_{0}} \\
& =-\left(a G u_{0}, \stackrel{\circ}{G} v\right)_{H_{1}}-\left(m u_{0}, v\right)_{H_{0}} .
\end{aligned}
$$

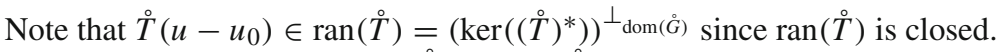

Now, let $v \in \operatorname{ker}\left(m^{*}-D a^{*} \stackrel{\circ}{G}\right)=\operatorname{ker}\left((\stackrel{\circ}{T})^{*}\right)$. Then,

$$
\begin{aligned}
0 & =-\left(\stackrel{\circ}{T}\left(u-u_{0}\right), v\right)_{\operatorname{dom}(\stackrel{\circ}{)})}=\left(a G u_{0}, \stackrel{\circ}{G} v\right)_{H_{1}}+\left(m u_{0}, v\right)_{H_{0}} \\
& =\left(G u_{0}, a^{*} \stackrel{\circ}{G} v\right)_{H_{1}}+\left(u_{0}, m^{*} v\right)_{H_{0}} \\
& =\left(G u_{0}, a^{*} \stackrel{\circ}{G} v\right)_{H_{1}}+\left(D G u_{0}, D a^{*} \stackrel{\circ}{G} v\right)_{H_{0}} \\
& =\left(G u_{0}, a^{*} \stackrel{\circ}{G} v\right)_{\operatorname{dom}(D)}=\left(G u_{0}, \pi_{\mathrm{BD}(D)} a^{*} \stackrel{\circ}{G} v\right)_{\mathrm{BD}(D)}
\end{aligned}
$$

as required.

' $\supset$ '. The proof is similar and for this inclusion it is essential that $\operatorname{ran}(\stackrel{\circ}{T})$ is closed. 
Corollary 5.4 Suppose that $\operatorname{ran}(\stackrel{\circ}{T})$ is closed in $\operatorname{dom}(\stackrel{\circ}{G})$. Then,

$$
\operatorname{dom}(\Lambda)=\left\{u_{0} \in \mathrm{BD}(G):\left(\Phi\left(\pi_{\mathrm{BD}(D)} a^{*} G v\right)\right)\left(u_{0}\right)=0 \text { for all } v \in \operatorname{ker}\left(m^{*}-D a^{*} \stackrel{\circ}{G}\right)\right\},
$$

where $\Phi: \mathrm{BD}(D) \rightarrow \mathrm{BD}(G)^{\prime}$ is the natural unitary map as in Proposition 2.8.

We emphasize that boundary regularity is not needed in Corollary 5.4.

The next lemma gives an easy-to-verify condition which implies that $\stackrel{\circ}{T}$ has closed range.

Lemma 5.5 If the inclusion $\tau: \operatorname{dom}(\stackrel{\circ}{G}) \rightarrow H_{0}$ is compact, then $\stackrel{\circ}{T}$ has closed range.

Proof There exist $\mu, \omega>0$ such that $\mu\|u\|_{\operatorname{dom}(\stackrel{\circ}{G})}^{2} \leqslant \operatorname{Re} \stackrel{\circ}{\mathfrak{b}}(u)+\omega\|\tau u\|_{H_{0}}^{2}$ for all $u \in \operatorname{dom}(\stackrel{\circ}{G})$.

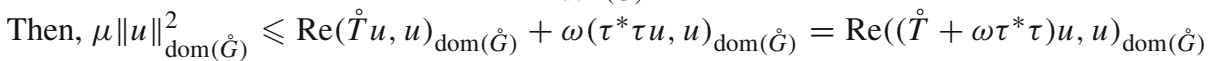
for all $u \in \operatorname{dom}(\stackrel{\circ}{G})$. So $\stackrel{\circ}{T}+\omega \tau^{*} \tau$ is injective and has closed range. Similarly, $(\stackrel{\circ}{T})^{*}+\omega \tau^{*} \tau$ is injective. So $\stackrel{\circ}{T}+\omega \tau^{*} \tau$ is invertible. Since $\omega \tau^{*} \tau$ is compact, the operator $\stackrel{\circ}{T}$ is Fredholm. In particular, the range of $\stackrel{\circ}{T}$ is closed.

Note that the operator $\tau$ is compact in the situation of Example 2.3.

Example 5.6 Let $\Omega \subset \mathbb{R}^{d}$ be a bounded Lipschitz domain with boundary $\Gamma$. Let $G$ and $D$ be as in Example 2.3. If $u_{0} \in \mathrm{BD}(G), v \in H_{0}$ and $\Phi: \operatorname{BD}(D) \rightarrow \mathrm{BD}(G)^{\prime}$ is the natural unitary map as in Proposition 2.8, then it follows from Example 2.10 and Proposition 2.9(b) that

$$
\begin{aligned}
\left(\Phi\left(\pi_{\mathrm{BD}(D)} a^{*} G v\right)\right)\left(u_{0}\right) & =\left\langle\left(v \pi_{\mathrm{BD}(D)} a^{*} G v\right), \operatorname{Tr} u_{0}\right\rangle_{\left(\operatorname{Tr} H^{1}(\Omega)\right)^{\prime} \times \operatorname{Tr} H^{1}(\Omega)} \\
& =\left\langle\left(v a^{*} G v\right), \operatorname{Tr} u_{0}\right\rangle_{\left(\operatorname{Tr} H^{1}(\Omega)\right)^{\prime} \times \operatorname{Tr} H^{1}(\Omega)} \\
& =\left\langle\left(\partial_{v}^{a^{*}} v\right), \operatorname{Tr} u_{0}\right\rangle_{H^{-1 / 2}(\partial \Omega), H^{1 / 2}(\partial \Omega)},
\end{aligned}
$$

where $\partial_{v}^{a^{*}}$ is the co-normal derivative. So Corollary 5.4 gives $\operatorname{dom}(\Lambda)=\left\{u_{0} \in \operatorname{BD}(G):\left\langle\left(\partial_{v}^{a^{*}} v\right), \operatorname{Tr} u_{0}\right\rangle_{H^{-1 / 2}(\partial \Omega), H^{1 / 2}(\partial \Omega)}=0\right.$ for all $\left.v \in \operatorname{ker}\left(m^{*}-D a^{*} \stackrel{\circ}{G}\right)\right\}$, in agreement with [8] Proposition 4.10.

Next, we turn to the Neumann-to-Dirichlet graph.

Proposition 5.7 Assume that $\operatorname{ran}(T)$ is closed in $\operatorname{dom}(G)$. Then,

$$
\operatorname{dom}\left(\Lambda^{-1}\right)=\left\{q_{0} \in \mathrm{BD}(D):\left(D q_{0}, \pi_{\mathrm{BD}(G)} v\right)_{\mathrm{BD}(G)}=0 \text { for all } v \in \operatorname{ker}\left(m^{*}-\stackrel{D}{D} a^{*} G\right)\right\} .
$$

Before we prove the latter proposition, we need a lemma.

Lemma 5.8 Let $q_{0} \in \mathrm{BD}(D)$. Let $f_{0} \in \operatorname{dom}(G)$ be such that

$$
\left(f_{0}, v\right)_{\operatorname{dom}(G)}=\left(D q_{0}, \pi_{\mathrm{BD}(G)} v\right)_{\mathrm{BD}(G)}
$$

for all $v \in \operatorname{dom}(G)$. Let $u \in \operatorname{dom}(G)$. Then, the following statements are equivalent.

(i) $T u=f_{0}$.

(ii) $u \in \operatorname{dom}(D a G), m u-D a G u=0$ and $q_{0}=\pi_{\mathrm{BD}(D)} a G u$.

Proof '(i) $\Rightarrow$ (ii)'. Let $v \in \operatorname{dom}(G)$. Then,

$$
\begin{aligned}
(m u, v)_{H_{0}}+(a G u, G v)_{H_{1}} & =\mathfrak{b}(u, v)=(T u, v)_{\operatorname{dom}(G)}=\left(f_{0}, v\right)_{\operatorname{dom}(G)} \\
& =\left(D q_{0}, \pi_{\mathrm{BD}(G)} v\right)_{\mathrm{BD}(G)} .
\end{aligned}
$$


Hence, $(m u, v)_{H_{0}}+(a G u, \stackrel{\circ}{G} v)_{H_{1}}=0$ for all $v \in \operatorname{dom}(\stackrel{\circ}{G})$. So $a G u \in \operatorname{dom}\left((\stackrel{\circ}{G})^{*}\right)=$ $\operatorname{dom}(D)$ and $D a G u=-(\stackrel{\circ}{G})^{*} a G u=m u$. In particular, $u \in \operatorname{dom}(D a G)$. Alternatively, if $v \in \mathrm{BD}(G)$, then

$$
\begin{aligned}
\left(D q_{0}, v\right)_{\mathrm{BD}(G)} & =\left(D q_{0}, \pi_{\mathrm{BD}(G)} v\right)_{\mathrm{BD}(G)}=(m u, v)_{H_{0}}+(a G u, G v)_{H_{1}} \\
& =(D a G u, D G v)_{H_{0}}+(a G u, G v)_{H_{1}}=(a G u, G v)_{\operatorname{dom}(D)} \\
& =\left(\pi_{\mathrm{BD}(D)} a G u, G v\right)_{\mathrm{BD}(D)}=\left(D \pi_{\mathrm{BD}(D)} a G u, v\right)_{\mathrm{BD}(G)}
\end{aligned}
$$

by Lemma 2.7. So $q_{0}=\pi_{\mathrm{BD}(D)} a G u$.

'(ii) $\Rightarrow(\mathrm{i})$ '. Let $v \in \operatorname{dom}(\stackrel{\circ}{G})$. Since $(\stackrel{\circ}{G})^{*}=-D$ one deduces that

$$
\begin{aligned}
(T u, v)_{\operatorname{dom}(G)} & =\mathfrak{b}(u, v)=(a G u, \stackrel{\circ}{G} v)_{H_{1}}+(m u, v)_{H_{0}} \\
& =-(D a G u, v)_{H_{0}}+(m u, v)_{H_{0}}=0=\left(D q_{0}, \pi_{\mathrm{BD}(G)} v\right)_{\mathrm{BD}(G)} \\
& =\left(f_{0}, v\right)_{\operatorname{dom}(G) .}
\end{aligned}
$$

Alternatively, if $v \in \mathrm{BD}(G)$, then

$$
\begin{aligned}
(T u, v)_{\operatorname{dom}(G)} & =\mathfrak{b}(u, v)=(a G u, G v)_{H_{1}}+(m u, v)_{H_{0}} \\
& =(a G u, G v)_{H_{1}}+(D a G u, D G v)_{H_{0}} \\
& =(a G u, G v)_{\operatorname{dom}(D)}=\left(\pi_{\mathrm{BD}(D)} a G u, G v\right)_{\mathrm{BD}(D)}=\left(q_{0}, G v\right)_{\mathrm{BD}(D)} \\
& =\left(D q_{0}, v\right)_{\mathrm{BD}(G)}=\left(f_{0}, v\right)_{\operatorname{dom}(G)} .
\end{aligned}
$$

So by linearity $(T u, v)_{\operatorname{dom}(G)}=\left(f_{0}, v\right)_{\operatorname{dom}(G)}$ for all $v \in \operatorname{dom}(G)$ and $T u=f_{0}$.

Proof of Proposition 5.7 Let $q_{0} \in \mathrm{BD}(D)$. Let $f_{0} \in \operatorname{dom}(G)$ be as in Lemma 5.8. Then, it follows from Lemma 5.8 that $q_{0} \in \operatorname{dom}\left(\Lambda^{-1}\right)$ if and only if $f_{0} \in \operatorname{ran}(T)$. But $\operatorname{ran}(T)=$ $\left(\operatorname{ker}\left(T^{*}\right)\right)^{\perp_{\text {dom }(G)}}$ since $\operatorname{ran}(T)$ is closed in $\operatorname{dom}(G)$. Now, $\operatorname{ker}\left(T^{*}\right)=\operatorname{ker}\left(m^{*}-\stackrel{\circ}{D} a^{*} G\right)$ because $G^{*}=-\stackrel{\circ}{D}$. Hence, $f_{0} \in \operatorname{ran}(T)$ if and only if $\left(D q_{0}, \pi_{\mathrm{BD}(G)} v\right)_{\mathrm{BD}(G)}=0$ for all $v \in \operatorname{ker}\left(m^{*}-\stackrel{D}{D} a^{*} G\right)$.

As in Lemma 5.5, one has the following sufficient condition for the closedness of $\operatorname{ran}(T)$.

Lemma 5.9 If the inclusion $\operatorname{dom}(G) \subset H_{0}$ is compact, then $\operatorname{ran}(T)$ is closed in $\operatorname{dom}(G)$.

In our model case Example 2.3, the inclusion $\operatorname{dom}(G) \subset H_{0}$ is compact if $\Omega$ is bounded and has a continuous boundary.

We conclude with a variant of the Dirichlet-to-Neumann graph involving an intermediate space as in Sect. 3. Throughout the remainder of this section, let $H$ be a Hilbert space and $\kappa \in \mathcal{L}(\mathrm{BD}(G), H)$ injective with dense range. Define

$$
\begin{array}{r}
\Lambda_{H}=\left\{(\varphi, \psi) \in H \times H \text { : there exists a } u_{0} \in \mathrm{BD}(G)\right. \text { such that } \\
\left.\kappa\left(u_{0}\right)=\varphi \text { and }\left(u_{0}, G \kappa^{*} \psi\right) \in \Lambda\right\} .
\end{array}
$$

We call $\Lambda_{H}$ the Dirichlet-to-Neumann graph in $H$ associated with $-D a G+m$. It follows from Lemma 5.2 that $\Lambda_{H}$ is single-valued if $\operatorname{ker}(m-D a \stackrel{\circ}{)})=\{0\}$.

The graph $\Lambda_{H}$ can be described with a form.

Proposition 5.10 Define $j: \operatorname{dom}(G) \rightarrow H$ by $j=\kappa \circ \pi_{\mathrm{BD}(G)}$. Then,

$\Lambda_{H}=\{(\varphi, \psi) \in H \times H$ : there exists a $u \in \operatorname{dom}(G)$ such that

$$
\left.j(u)=\varphi \text { and } \mathfrak{b}(u, v)=(\psi, j(v))_{\operatorname{dom}(G)} \text { for all } v \in \operatorname{dom}(G)\right\} .
$$


Proof This follows as in the proof of Proposition 3.6.

Corollary 5.11 If $\operatorname{ker}(-D a \stackrel{\circ}{G}+m)=\{0\}$ and the inclusion $\operatorname{dom}(G) \subset H_{0}$ is compact, then $\Lambda_{H}$ is an m-sectorial operator.

Proof Let $j=\kappa \circ \pi_{\mathrm{BD}(G)}: \operatorname{dom}(G) \rightarrow H$ and let $V(\mathfrak{b})=\{u \in \operatorname{dom}(G): \mathfrak{b}(u, v)=$ 0 for all $v \in \operatorname{ker} j\}$. Then, $V(\mathfrak{b}) \cap \operatorname{ker} j=\operatorname{ker}(-D a \stackrel{\circ}{G}+m)=\{0\}$. Then, the statement follows from [1] Theorem 8.11 and Proposition 5.10.

Even if the inclusion $\operatorname{dom}(G) \subset H_{0}$ is compact, then in general $\Lambda_{H}$ is not an m-sectorial graph. A counterexample has been given in [7] Example 3.7.

\section{Resolvent convergence, non-coercive case}

In this section, we consider resolvent convergence of a sequence of Dirichlet-to-Neumann operators without the coercivity condition on $m$. Throughout this section, we adopt the notation and assumptions as in the beginning of Sect. 2. Let $H$ be a Hilbert space, and let $\kappa \in \mathcal{L}(\mathrm{BD}(G), H)$ be one-to-one with dense range. Set $j=\kappa \circ \pi_{\mathrm{BD}(G)}: \operatorname{dom}(G) \rightarrow H$.

We need a stronger version of convergence for the leading coefficients, which we next introduce. Let $a, a_{1}, a_{2}, \ldots \in \mathcal{L}\left(H_{1}\right)$ be coercive. We say that $\left(a_{n}\right)_{n \in \mathbb{N}}$ converges to a independent of the boundary conditions if for every strictly increasing sequence $\left(n_{k}\right)_{k \in \mathbb{N}}$ in $\mathbb{N}$, all $f, f_{1}, f_{2}, \ldots \in H_{0}$ and all $u, u_{1}, u_{2}, \ldots \in \operatorname{dom}(G)$ with

$$
\left[\begin{array}{l}
\lim _{k \rightarrow \infty} f_{k}=f \text { weakly in } H_{0}, \\
\lim _{k \rightarrow \infty} u_{k}=u \text { weakly in } \operatorname{dom}(G), \text { and } \\
u_{k} \in \operatorname{dom}\left(D a_{n_{k}} G\right) \text { and }-D a_{n_{k}} G u_{k}=f_{k} \text { for all } k \in \mathbb{N}
\end{array}\right.
$$

it follows that $\lim _{k \rightarrow \infty} a_{n_{k}} G u_{k}=a G u$ weakly in $H_{1}$.

Note that $D$ is weakly closed and $\lim _{k \rightarrow \infty} D\left(a_{n_{k}} G u_{k}\right)=\lim _{k \rightarrow \infty}-f_{k}=-f$ weakly in $H_{0}$. So $a G u \in \operatorname{dom}(D)$ and $-D a G u=f$. In particular, $u \in \operatorname{dom}(D a G)$.

Example 6.1 In this example, we show that in the classical situation, convergence of the coefficients independent of the boundary conditions is equivalent to the already studied notion of $H$-convergence, see [15] and [9].

Let $\Omega \subset \mathbb{R}^{d}$ be open and bounded. Further, let $H_{0}, H_{1}, G$ and $D$ be as in Example 2.3. We identify an element of $L_{\infty}\left(\Omega, \mathbb{C}^{d \times d}\right)$ with an element of $\mathcal{L}\left(H_{1}\right)$ in the natural way. Let $a, a_{1}, a_{2}, \ldots \in L_{\infty}\left(\Omega, \mathbb{R}^{d \times d}\right)$. Note that we require that the matrices are real valued, but they do not have to be symmetric. Suppose that $\operatorname{Re} a_{n} \geqslant \mu I$ for all $n \in \mathbb{N}, \operatorname{Re} a \geqslant \mu I$ and $\sup _{n}\left\|a_{n}\right\|_{\mathcal{L}\left(H_{1}\right)}<\infty$.

Recall that the sequence $\left(a_{n}\right)_{n \in \mathbb{N}}$ is called $H$-convergent to $a$, if for all $f \in H^{-1}(\Omega)$ and for all $n \in \mathbb{N}$ with $u_{n} \in H_{0}^{1}(\Omega)$ satisfying

$$
\left(a_{n} \operatorname{grad} u_{n}, \operatorname{grad} v\right)_{L_{2}(\Omega)^{d}}=f(v)
$$

for all $v \in H_{0}^{1}(\Omega)$, it follows that $\lim _{n \rightarrow \infty} u_{n}=u$ weakly in $H_{0}^{1}(\Omega)$ and $\lim _{n \rightarrow \infty} a_{n} \operatorname{grad} u_{n}$ $=a \operatorname{grad} u$ weakly in $L_{2}(\Omega)^{d}$, where $u \in H_{0}^{1}(\Omega)$ is such that

$$
(a \operatorname{grad} u, \operatorname{grad} v)_{L_{2}(\Omega)^{d}}=f(v)
$$

for all $v \in H_{0}^{1}(\Omega)$. 
Suppose that the sequence $\left(a_{n}\right)_{n \in \mathbb{N}}$ is $H$-convergent to $a$. We show that $\left(a_{n}\right)_{n \in \mathbb{N}}$ converges to $a$ independent of the boundary conditions. Let $f, f_{1}, f_{2}, \ldots \in L_{2}(\Omega), u, u_{1}, u_{2}, \ldots \in$ $H^{1}(\Omega)$ and $\left(n_{k}\right)_{k \in \mathbb{N}}$ satisfy (11). Then, every subsequence $\left(a_{n_{k}}\right)_{k \in \mathbb{N}}$ is $H$-convergent to $a$ by the discussion after Definition 6.4 in [15]. So without loss of generality we may assume that $n_{k}=k$ for all $k \in \mathbb{N}$. As $\left(u_{k}\right)_{k \in \mathbb{N}}$ converges to $u$ weakly in $H^{1}(\Omega)$, it also converges weakly in $H_{\text {loc }}^{1}(\Omega)$. The inclusion $H_{0}^{1}(\Omega) \subset L_{2}(\Omega)$ is compact since $\Omega$ is bounded. Hence, also the inclusion $L_{2}(\Omega) \subset\left(H_{0}^{1}(\Omega)\right)^{\prime}=H^{-1}(\Omega)$ is compact. Therefore, $\left(f_{k}\right)_{k \in \mathbb{N}}$ converges strongly to $f$ in $H^{-1}(\Omega) \subset H_{\mathrm{loc}}^{-1}(\Omega)$. Then, the criteria of Lemma 10.3 in [15] are fulfilled and we obtain that $\left(a_{k} G u_{k}\right)_{k \in \mathbb{N}}$ converges weakly to $a G u$ in $L_{2, \text { loc }}(\Omega)^{d}$. Since the sequence $\left(a_{k} G u_{k}\right)_{k \in \mathbb{N}}$ in $L_{2}(\Omega)^{d}$ is bounded in $L_{2}(\Omega)^{d}$, there exists a $q \in L_{2}(\Omega)^{d}$ and a subsequence of $\left(a_{k} G u_{k}\right)_{k \in \mathbb{N}}$ that weakly converges to $q$ in $L_{2}(\Omega)^{d}$. By uniqueness of limits in $L_{2, \operatorname{loc}}(\Omega)^{d}$, we must have that $q=a G u$. So the subsequence converges to $a G u$ in $L_{2}(\Omega)^{d}$. Using the standard subsequence argument, we deduce that $\left(a_{k} G u_{k}\right)_{k \in \mathbb{N}}$ converges weakly to $a G u$ in $L_{2}(\Omega)^{d}=H_{1}$.

Conversely, suppose that $\left(a_{n}\right)_{n \in \mathbb{N}}$ converges to $a$ independent of the boundary conditions. We shall prove that the sequence $\left(a_{n}\right)_{n \in \mathbb{N}}$ is $H$-convergent to $a$. Let $f \in H^{-1}(\Omega)$. For all $n \in \mathbb{N}$, let $u_{n}, u \in H_{0}^{1}(\Omega)$ satisfy

$$
\left(a_{n} \operatorname{grad} u_{n}, \operatorname{grad} v\right)_{L_{2}(\Omega)^{d}}=f(v) \quad \text { and } \quad(a \operatorname{grad} u, \operatorname{grad} v)_{L_{2}(\Omega)^{d}}=f(v)
$$

for all $v \in H_{0}^{1}(\Omega)$. We need to show that $\lim u_{n}=u$ weakly in $H_{0}^{1}(\Omega)$ and $\lim a_{n} \operatorname{grad} u_{n}=$ $a$ grad $u$ weakly in $L_{2}(\Omega)^{d}$. Since $L_{2}(\Omega)$ is dense in $H^{-1}(\Omega)$, there exists a sequence $\left(f_{\ell}\right)_{\ell \in \mathbb{N}}$ in $L_{2}(\Omega)$ such that $\lim _{\ell \rightarrow \infty} f_{\ell}=f$ in $H^{-1}(\Omega)$. For all $n, \ell \in \mathbb{N}$, let $u_{n}^{\ell}, u^{\ell} \in H_{0}^{1}(\Omega)$ be such that

$\left(a_{n} \operatorname{grad} u_{n}^{\ell}, \operatorname{grad} v\right)_{L_{2}(\Omega)^{d}}=\left(f_{\ell}, v\right)_{L_{2}(\Omega)} \quad$ and $\left(a \operatorname{grad} u^{\ell}, \operatorname{grad} v\right)_{L_{2}(\Omega)^{d}}=\left(f_{\ell}, v\right)_{L_{2}(\Omega)}$

for all $v \in H_{0}^{1}(\Omega)$.

Let $\ell \in \mathbb{N}$. We shall show that $\lim _{n \rightarrow \infty} u_{n}^{\ell}=u^{\ell}$ weakly in $H_{0}^{1}(\Omega)$ and $\lim _{n \rightarrow \infty} a_{n} \operatorname{grad} u_{n}^{\ell}$ $=a \operatorname{grad} u^{\ell}$ weakly in $L_{2}(\Omega)^{d}$. Note that the sequence $\left(u_{n}^{\ell}\right)_{n \in \mathbb{N}}$ is bounded in $H_{0}^{1}(\Omega)$. Choose $\left(n_{k}\right)_{k \in \mathbb{N}}$ to be a strictly increasing sequence of natural numbers such that $\left(u_{n_{k}}^{\ell}\right)_{k \in \mathbb{N}}$ weakly converges in $H_{0}^{1}(\Omega)$, say to $w^{\ell} \in H_{0}^{1}(\Omega)$. Since the sequence $\left(a_{n}\right)_{n \in \mathbb{N}}$ converges to $a$ independent of the boundary conditions, one deduces that $\lim _{k \rightarrow \infty} a_{n_{k}} \operatorname{grad} u_{n_{k}}^{\ell}=a \operatorname{grad} w^{\ell}$ weakly in $L_{2}(\Omega)^{d}$ and

$$
\left(a \operatorname{grad} w^{\ell}, \operatorname{grad} v\right)_{L_{2}(\Omega)^{d}}=\left(f_{\ell}, v\right)_{L_{2}(\Omega)}
$$

for all $v \in H_{0}^{1}(\Omega)$. Uniqueness of $u^{\ell}$ implies $w^{\ell}=u^{\ell}$. Hence, $\lim _{n \rightarrow \infty} u_{n}^{\ell}=u^{\ell}$ weakly in $H_{0}^{1}(\Omega)$ by a subsubsequence argument. Since the sequence $\left(a_{n}\right)_{n \in \mathbb{N}}$ converges to $a$ independent of the boundary conditions, we obtain $\lim _{n \rightarrow \infty} a_{n} \operatorname{grad} u_{n}^{\ell}=a \operatorname{grad} u^{\ell}$ weakly in $L_{2}(\Omega)^{d}$.

By the Dirichlet-type Poincaré inequality, there exists a $c_{0}>0$ such that $\|v\|_{L_{2}(\Omega)} \leqslant$ $c_{0}\|\operatorname{grad} v\|_{L_{2}(\Omega)^{d}}$ for all $v \in H_{0}^{1}(\Omega)$. If $n, \ell \in \mathbb{N}$, then

$$
\begin{aligned}
\mu\left\|\operatorname{grad}\left(u_{n}^{\ell}-u_{n}\right)\right\|_{L_{2}(\Omega)^{d}}^{2} & \leqslant \operatorname{Re}\left(a_{n} \operatorname{grad}\left(u_{n}^{\ell}-u_{n}\right), \operatorname{grad}\left(u_{n}^{\ell}-u_{n}\right)\right)_{L_{2}(\Omega)^{d}} \\
& =\operatorname{Re}\left(f_{\ell}-f\right)\left(u_{n}^{\ell}-u_{n}\right) \\
& \leqslant\left\|f_{\ell}-f\right\|_{H^{-1}(\Omega)}\left\|u_{n}^{\ell}-u_{n}\right\|_{H_{0}^{1}(\Omega)} \\
& \leqslant\left(1+c_{0}\right)\left\|f_{\ell}-f\right\|_{H^{-1}(\Omega)}\left\|\operatorname{grad}\left(u_{n}^{\ell}-u_{n}\right)\right\|_{L_{2}(\Omega)^{d}} .
\end{aligned}
$$


Therefore,

$$
\left\|u_{n}^{\ell}-u_{n}\right\|_{H_{0}^{1}(\Omega)} \leqslant\left(1+c_{0}\right)\left\|\operatorname{grad}\left(u_{n}^{\ell}-u_{n}\right)\right\|_{L_{2}(\Omega)^{d}} \leqslant\left(1+c_{0}\right)^{2} \mu^{-1}\left\|f_{\ell}-f\right\|_{H^{-1}(\Omega)} .
$$

Similarly, $\left\|u^{\ell}-u\right\|_{H_{0}^{1}(\Omega)} \leqslant\left(1+c_{0}\right)^{2} \mu^{-1}\left\|f_{\ell}-f\right\|_{H^{-1}(\Omega)}$. If $n, \ell \in \mathbb{N}$, then $\left\|a_{n} \operatorname{grad} u_{n}^{\ell}-a_{n} \operatorname{grad} u_{n}\right\|_{L_{2}(\Omega)^{d}} \leqslant c\left\|u_{n}^{\ell}-u_{n}\right\|_{H_{0}^{1}(\Omega)} \leqslant c\left(1+c_{0}\right)^{2} \mu^{-1}\left\|f_{\ell}-f\right\|_{H^{-1}(\Omega)}$,

where $c=\|a\|_{\mathcal{L}\left(H_{1}\right)} \vee \sup _{k \in \mathbb{N}}\left\|a_{k}\right\|_{\mathcal{L}\left(H_{1}\right)}$. Similarly, one deduces $\left\|a \operatorname{grad} u^{\ell}-a \operatorname{grad} u\right\|_{L_{2}(\Omega)^{d}}$ $\leqslant c\left(1+c_{0}\right)^{2} \mu^{-1}\left\|f_{\ell}-f\right\|_{H^{-1}(\Omega)}$. Now, let $v \in H_{0}^{1}(\Omega)$. Then,

$$
\begin{aligned}
\left|\left(u_{n}-u, v\right)_{H_{0}^{1}(\Omega)}\right| \leqslant & \left|\left(u_{n}-u_{n}^{\ell}, v\right)_{H_{0}^{1}(\Omega)}\right|+\left|\left(u_{n}^{\ell}-u^{\ell}, v\right)_{H_{0}^{1}(\Omega)}\right|+\left|\left(u^{\ell}-u, v\right)_{H_{0}^{1}(\Omega)}\right| \\
\leqslant & \left(1+c_{0}\right)^{2} \mu^{-1}\left\|f-f_{\ell}\right\|_{H^{-1}(\Omega)}\|v\|_{H_{0}^{1}(\Omega)}+\left|\left(u_{n}^{\ell}-u^{\ell}, v\right)_{H_{0}^{1}(\Omega)}\right| \\
& +\left(1+c_{0}\right)^{2} \mu^{-1}\left\|f-f_{\ell}\right\|_{H^{-1}(\Omega)}\|v\|_{H_{0}^{1}(\Omega)}
\end{aligned}
$$

for all $\ell, n \in \mathbb{N}$, which yields $\lim _{n \rightarrow \infty} u_{n}=u$ weakly in $H_{0}^{1}(\Omega)$. It follows similarly that $\lim _{n \rightarrow \infty} a_{n} \operatorname{grad} u_{n}=a \operatorname{grad} u$ weakly in $L_{2}(\Omega)^{d}$. Hence, the sequence $\left(a_{n}\right)_{n \in \mathbb{N}}$ is $H$-convergent to $a$.

The condition $\left(a_{n}\right)_{n \in \mathbb{N}}$ converges to $a$ independent of the boundary conditions, which we use in this section, is stronger than the condition used for the convergence in Theorem 4.2.

Proposition 6.2 Let $a, a_{1}, a_{2}, \ldots \in \mathcal{L}\left(H_{1}\right)$ and $\mu>0$. Suppose that $\operatorname{Re} a_{n} \geqslant \mu I$ for all $n \in \mathbb{N}$ and $\operatorname{Re} a \geqslant \mu I$. Suppose that $\left(a_{n}\right)_{n \in \mathbb{N}}$ converges to a independent of the boundary conditions. Further assume that the inclusion $\operatorname{dom}(G) \subset H_{0}$ is compact. Let $\iota: \operatorname{ran}(G) \hookrightarrow$ $H_{1}$ be the embedding map. Then, $\lim _{n \rightarrow \infty}\left(\iota^{*} a_{n} \iota\right)^{-1}=\left(\iota^{*} a \iota\right)^{-1}$ in the weak operator topology on $\mathcal{L}(\operatorname{ran}(G))$.

Proof Let $q \in \operatorname{ran}(G) \cap \operatorname{dom}(D)$. Let $n \in \mathbb{N}$. Write $r_{n}=\left(\iota^{*} a_{n} \iota\right)^{-1} q$. Then, $r_{n} \in \operatorname{ran}(G)$ and $\left\|r_{n}\right\|_{H_{1}} \leqslant \mu^{-1}\|q\|_{H_{1}}$. There exists a $u_{n} \in \operatorname{dom}(G) \cap(\operatorname{ker}(G))^{\perp_{H_{0}}}$ such that $G u_{n}=r_{n}$. Then, the sequence $\left(u_{n}\right)_{n \in \mathbb{N}}$ is bounded in dom $G$ by Lemma 4.1(a). Passing to a subsequence if necessary, there exists a $u \in \operatorname{dom}(G) \operatorname{such}$ that $\lim u_{n}=u$ weakly in $\operatorname{dom}(G)$. Let $n \in \mathbb{N}$. Then, $q=\iota^{*} a_{n} \iota r_{n}=\iota^{*} a_{n} G u_{n}$. Since $q \in \operatorname{dom}(\stackrel{D}{D})$ it follows from Lemma 4.4(a) that $a_{n} G u_{n} \in \operatorname{dom}(\stackrel{\circ}{D})$ and $\stackrel{\circ}{D} a_{n} G u_{n}=\stackrel{\circ}{D}^{*} \iota_{n} G u_{n}=\stackrel{\circ}{D} q$. Because $\left(a_{n}\right)_{n \in \mathbb{N}}$ converges to $a$ independent of the boundary conditions, we obtain that $\lim a_{n} G u_{n}=a G u$ weakly in $H_{1}$. Since the operator $\stackrel{\circ}{D}$ is closed, we obtain that $a G u \in \operatorname{dom}(\stackrel{\circ}{D})$ and $\stackrel{\circ}{D} a G u=\stackrel{\circ}{D} q$. Using again Lemma 4.4(a), one deduces that $\iota^{*} a G u \in \operatorname{dom}(\stackrel{\circ}{D})$ and $\stackrel{\circ}{D}^{*} a G u=\stackrel{\circ}{D} q$. Hence,

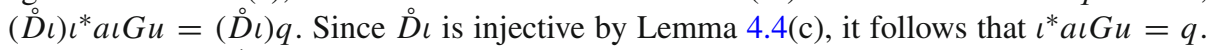
So $G u=\left(\iota^{*} a \iota\right)^{-1} q$. Then,

$$
\lim _{n \rightarrow \infty}\left(\iota^{*} a_{n} \iota\right)^{-1} q=\lim _{n \rightarrow \infty} r_{n}=\lim _{n \rightarrow \infty} G u_{n}=G u=\left(\iota^{*} a \iota\right)^{-1} q
$$

weakly in $\operatorname{ran}(G)$.

Finally, since $\sup \left\|\left(\iota^{*} a_{n} \iota\right)^{-1}\right\|_{\mathcal{L}(\operatorname{ran}(G))}<\infty$ and $\operatorname{ran}(G) \cap \operatorname{dom}(\stackrel{D}{)}$ is dense in $\operatorname{ran}(G)$ by Lemma 4.4(b), one concludes that $\lim \left(\iota^{*} a_{n} \iota\right)^{-1}=\left(\iota^{*} a \iota\right)^{-1}$ in the weak operator topology on $\mathcal{L}(\operatorname{ran}(G))$.

Remark 6.3 The above proposition is also valid if $\iota$ is replaced by the embedding of a closed subspace of $\operatorname{ran}(G)$ which contains $\operatorname{ran}(\stackrel{\circ}{G})$. This is the motivation for the terminology $\left(a_{n}\right)_{n \in \mathbb{N}}$ converges to $a$ independent of the boundary conditions.

The main theorem of this section is as follows. 
Theorem 6.4 Let $a, a_{1}, a_{2}, \ldots \in \mathcal{L}\left(H_{1}\right), m, m_{1}, m_{2}, \ldots \in \mathcal{L}\left(H_{0}\right)$ and $\mu>0$. Suppose that $\operatorname{Re} a_{n} \geqslant \mu I$ for all $n \in \mathbb{N}, \operatorname{Re} a \geqslant \mu I$ and $\sup _{n}\left\|a_{n}\right\|_{\mathcal{L}\left(H_{1}\right)}<\infty$. Suppose that $\left(a_{n}\right)_{n \in \mathbb{N}}$ converges to a independent of the boundary conditions and $\lim m_{n}=m$ in the weak operator topology on $\mathcal{L}\left(H_{0}\right)$. Assume that $\operatorname{ker}\left(m_{n}-D a_{n} \stackrel{\circ}{G}\right)=\{0\}$ for all $n \in \mathbb{N}$ and $\operatorname{ker}(m-D a \stackrel{G}{)})=\{0\}$. Further assume that the inclusion $\operatorname{dom}(G) \subset H_{0}$ is compact.

For all $n \in \mathbb{N}$, let $\Lambda_{H}^{(n)}$ and $\Lambda_{H}$ be the Dirichlet-to-Neumann operators in $H$ associated with $-D a_{n} G+m_{n}$ and $-D a G+m$, respectively. Then, one has the following.

(a) The sequence $\left(\Lambda_{H}^{(n)}\right)_{n \in \mathbb{N}}$ of operators is uniformly sectorial.

(b) $\lim _{n \rightarrow \infty}\left(\lambda I+\Lambda_{H}^{(n)}\right)^{-1}=\left(\lambda I+\Lambda_{H}\right)^{-1}$ in the weak operator topology for all large $\lambda>0$.

(c) If $\kappa$ is compact, then

$$
\lim _{n \rightarrow \infty}\left(\lambda I+\Lambda_{H}^{(n)}\right)^{-1}=\left(\lambda I+\Lambda_{H}\right)^{-1}
$$

uniformly in $\mathcal{L}(H)$ for all large $\lambda>0$.

The proof requires a lot of preparation. Adopt the notation and assumptions of Theorem 6.4. For all $n \in \mathbb{N}$, define $\mathfrak{b}_{n}: \operatorname{dom}(G) \times \operatorname{dom}(G) \rightarrow \mathbb{C}$ by

$$
\mathfrak{b}_{n}(u, v)=\left(a_{n} G u, G v\right)_{H_{1}}+\left(m_{n} u, v\right)_{H_{0}}
$$

and define $V\left(\mathfrak{b}_{n}\right)=\left\{u \in \operatorname{dom}(G): \mathfrak{b}_{n}(u, v)=0\right.$ for all $v \in$ ker $\left.j\right\}$. Define similarly $\mathfrak{b}$ and $V(\mathfrak{b})$.

Lemma 6.5 For all $\varepsilon>0$, there exists an $\omega>0$ such that

$$
\|u\|_{H_{0}}^{2} \leqslant \varepsilon\|u\|_{\operatorname{dom}(G)}^{2}+\omega\|j(u)\|_{H}^{2}
$$

for all $n \in \mathbb{N}$ and $u \in V\left(\mathfrak{b}_{n}\right)$.

Proof Let $n \in \mathbb{N}$. Since $\operatorname{ker}\left(m_{n}-D a_{n} \stackrel{\circ}{G}\right)=\{0\}$, the restriction $\left.j\right|_{V\left(\mathfrak{b}_{n}\right)}$ is injective. Because also the inclusion $\operatorname{dom}(G) \subset H_{0}$ is compact, it follows that for all $\varepsilon>0$ there exists an $\omega>0$ such that

$$
\|u\|_{H_{0}}^{2} \leqslant \varepsilon\|u\|_{\operatorname{dom}(G)}^{2}+\omega\|j(u)\|_{H}^{2}
$$

for all $u \in V\left(\mathfrak{b}_{n}\right)$. We next show that one can choose $\omega$ uniformly in $n$.

Suppose the lemma is false. Then, without loss of generality and passing to a subsequence if necessary there exist $\varepsilon>0$ and for all $n \in \mathbb{N}$ there exists a $u_{n} \in V\left(\mathfrak{b}_{n}\right)$ such that

$$
\left\|u_{n}\right\|_{H_{0}}^{2}>\varepsilon\left\|u_{n}\right\|_{\operatorname{dom}(G)}^{2}+n\left\|j\left(u_{n}\right)\right\|_{H}^{2} .
$$

Without loss of generality, we may assume that $\left\|u_{n}\right\|_{H_{0}}=1$ for all $n \in \mathbb{N}$. Then, $\varepsilon\left\|u_{n}\right\|_{\operatorname{dom}(G)}^{2} \leqslant 1$ for all $n \in \mathbb{N}$, so the sequence $\left(u_{n}\right)_{n \in \mathbb{N}}$ is bounded in $\operatorname{dom}(G)$. Passing to a subsequence if necessary there exists a $u \in \operatorname{dom}(G)$ such that $\lim u_{n}=u$ weakly in $\operatorname{dom}(G)$. Since the inclusion $\operatorname{dom}(G) \subset H_{0}$ is compact, it follows that $u=\lim u_{n}$ in $H_{0}$. In particular, $\|u\|_{H_{0}}=1$ and $u \neq 0$. Also, $j(u)=\lim j\left(u_{n}\right)=0$ in $H$, $\operatorname{so} u \in \operatorname{ker} j=\operatorname{dom}(\stackrel{\circ}{G})$.

If $n \in \mathbb{N}$, then $\left(a_{n} G u_{n}, \stackrel{\circ}{G} v\right)_{H_{1}}=-\left(m_{n} u_{n}, v\right)_{H_{0}}$ for all $v \in \operatorname{dom}(\stackrel{\circ}{G})=\operatorname{ker} j$, since $u_{n} \in V\left(\mathfrak{b}_{n}\right)$. Therefore, $a_{n} G u_{n} \in \operatorname{dom}\left((\stackrel{\circ}{G})^{*}\right)=\operatorname{dom}(D)$ and $-D a_{n} G u_{n}=-m_{n} u_{n}$. Next, $\lim m_{n} u_{n}=m u$ weakly in $H_{0}$. Since $\left(a_{n}\right)_{n \in \mathbb{N}}$ converges to $a$ independent of the boundary conditions one deduces that $a G u \in \operatorname{dom}(D)$ and $-D a G u=-m u$. Then, $u \in$ $\operatorname{ker}(m-D a \stackrel{\circ}{)})=\{0\}$. So $u=0$. This is a contradiction. 
Lemma 6.6 There exist $\tilde{\mu}, \omega>0$ such that

$$
\tilde{\mu}\|u\|_{\operatorname{dom}(G)}^{2} \leqslant \operatorname{Re} \mathfrak{b}_{n}(u)+\omega\|j(u)\|_{H}^{2}
$$

for all $n \in \mathbb{N}$ and $u \in V\left(\mathfrak{b}_{n}\right)$.

Proof Let $\tilde{\omega}=\mu+\sup _{n}\left\|m_{n}\right\|_{\mathcal{L}\left(H_{0}\right)}$. Then,

$$
\mu\|u\|_{\operatorname{dom}(G)}^{2} \leqslant \operatorname{Re}\left(a_{n} G u, G u\right)_{H_{1}}+\mu\|u\|_{H_{0}}^{2} \leqslant \operatorname{Re} \mathfrak{b}_{n}(u)+\tilde{\omega}\|u\|_{H_{0}}^{2}
$$

for all $n \in \mathbb{N}$ and $u \in \operatorname{dom}(G)$.

Choose $\varepsilon=\frac{\mu}{2 \tilde{\omega}}$ and let $\omega>0$ be as in Lemma 6.5. Let $n \in \mathbb{N}$ and $u \in V\left(\mathfrak{b}_{n}\right)$. Then,

$$
\begin{aligned}
\mu\|u\|_{\operatorname{dom}(G)}^{2} & \leqslant \operatorname{Re} \mathfrak{b}_{n}(u)+\tilde{\omega}\|u\|_{H_{0}}^{2} \\
& \leqslant \operatorname{Re} \mathfrak{b}_{n}(u)+\tilde{\omega}\left(\frac{\mu}{2 \tilde{\omega}}\|u\|_{\operatorname{dom}(G)}^{2}+\omega\|j(u)\|_{H}^{2}\right) \\
& =\operatorname{Re} \mathfrak{b}_{n}(u)+\frac{\mu}{2}\|u\|_{\operatorname{dom}(G)}^{2}+\omega \tilde{\omega}\|j(u)\|_{H}^{2} .
\end{aligned}
$$

So

$$
\frac{\mu}{2}\|u\|_{\operatorname{dom}(G)}^{2} \leqslant \operatorname{Re} \mathfrak{b}_{n}(u)+\omega \tilde{\omega}\|j(u)\|_{H}^{2}
$$

and the lemma follows.

Now, we are able to prove Theorem 6.4.

Proof of Theorem 6.4 Let $\tilde{\mu}, \omega>0$ be as in Lemma 6.6.

'(a)'. Set $c=\sup _{n \in \mathbb{N}}\left(\left\|a_{n}\right\|_{\mathcal{L}\left(H_{1}\right)}+\left\|m_{n}\right\|_{\mathcal{L}\left(H_{0}\right)}\right)$. Let $n \in \mathbb{N}$ and $\varphi \in \operatorname{dom}\left(\Lambda_{H}^{(n)}\right)$. There exists a $u \in \operatorname{dom}(G)$ such that $j(u)=\varphi$ and $\mathfrak{b}_{n}(u, v)=\left(\Lambda_{H}^{(n)} \varphi, j(v)\right)_{H}$ for all $v \in \operatorname{dom}(G)$. Then, $u \in V\left(\mathfrak{b}_{n}\right)$ and $\left(\left(\Lambda_{H}^{(n)}+\omega I\right) \varphi, \varphi\right)_{H}=\mathfrak{b}_{n}(u)+\omega\|j(u)\|_{H}^{2}, \operatorname{so} \operatorname{Re}\left(\left(\Lambda_{H}^{(n)}+\omega I\right) \varphi, \varphi\right)_{H} \geqslant$ $\tilde{\mu}\|u\|_{\operatorname{dom}(G)}^{2}$. Therefore,

$$
\left|\operatorname{Im}\left(\left(\Lambda_{H}^{(n)}+\omega I\right) \varphi, \varphi\right)_{H}\right|=\left|\operatorname{Im} \mathfrak{b}_{n}(u)\right| \leqslant c\|u\|_{\operatorname{dom}(G)}^{2} \leqslant \frac{c}{\tilde{\mu}} \operatorname{Re}\left(\left(\Lambda_{H}^{(n)}+\omega I\right) \varphi, \varphi\right)_{H} .
$$

Hence, the operators $\Lambda_{H}^{(n)}$ are sectorial with vertex $-\omega$ and semi-angle $\arctan \frac{c}{\tilde{\mu}}$, uniformly in $n$.

'(b)'. In order not to repeat part of the proof in Statement (c), we first prove something more general. Let $\lambda>\omega$. Let $\psi, \psi_{1}, \psi_{2}, \ldots \in H$ and suppose that $\lim \psi_{n}=\psi$ weakly in $H$. We shall prove that $\lim \left(\lambda I+\Lambda_{H}^{(n)}\right)^{-1} \psi_{n}=\left(\lambda I+\Lambda_{H}\right)^{-1} \psi$ weakly in $H$.

Let $n \in \mathbb{N}$. Set $\varphi_{n}=\left(\lambda I+\Lambda_{H}^{(n)}\right)^{-1} \psi_{n}$. There exists a $u_{n} \in V\left(\mathfrak{b}_{n}\right)$ such that $j\left(u_{n}\right)=\varphi_{n}$ and

$$
\mathfrak{b}_{n}\left(u_{n}, v\right)+\lambda\left(j\left(u_{n}\right), j(v)\right)_{H}=\left(\psi_{n}, j(v)\right)_{H}
$$

for all $v \in \operatorname{dom}(G)$. Choose $v=u_{n}$. Then, Lemma 6.6 gives

$$
\begin{aligned}
\tilde{\mu}\left\|u_{n}\right\|_{\operatorname{dom}(G)}^{2} & \leqslant \operatorname{Re} \mathfrak{b}_{n}\left(u_{n}\right)+\lambda\left\|j\left(u_{n}\right)\right\|_{H}^{2}=\operatorname{Re}\left(\psi_{n}, j\left(u_{n}\right)\right)_{H} \\
& \leqslant\left\|\psi_{n}\right\|_{H}\|j\|_{\mathcal{L}(\operatorname{dom}(G), H)}\left\|u_{n}\right\|_{\operatorname{dom}(G)} .
\end{aligned}
$$

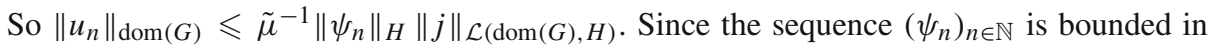
$H$, the sequence $\left(u_{n}\right)_{n \in \mathbb{N}}$ is bounded in $\operatorname{dom}(G)$. Passing to a subsequence if necessary, there exists a $u \in \operatorname{dom}(G)$ such that $\lim u_{n}=u$ weakly in $\operatorname{dom}(G)$. Since the inclusion $\operatorname{dom}(G) \subset H_{0}$ is compact, one deduces that $\lim u_{n}=u$ in $H_{0}$. Then, $\lim m_{n} u_{n}=m u$ 
weakly in $H_{0}$. Moreover, $\lim \varphi_{n}=\lim j\left(u_{n}\right)=j(u)$ weakly in $H$. Next, we show that $j(u)=\left(\lambda I+\Lambda_{H}\right)^{-1} \psi$.

Let $n \in \mathbb{N}$. If $v \in \operatorname{ker} j=\operatorname{dom}(\stackrel{\circ}{G})$, then $\mathfrak{b}_{n}\left(u_{n}, v\right)=0$, so $\left(a_{n} G u_{n}, \stackrel{\circ}{G} v\right)_{H_{1}}=$ $-\left(m_{n} u_{n}, v\right)_{H_{0}}$. Hence, $a_{n} G u_{n} \in \operatorname{dom}\left((\stackrel{\circ}{G})^{*}\right)=\operatorname{dom}(D)$ and $-D a_{n} G u_{n}=-m_{n} u_{n}$. In particular, $u_{n} \in \operatorname{dom}\left(D a_{n} G\right)$. Moreover, $\lim u_{n}=u$ weakly in $\operatorname{dom}(G)$ and $\lim m_{n} u_{n}=m u$ weakly in $H_{0}$. Since $\left(a_{n}\right)_{n \in \mathbb{N}}$ converges to $a$ independent of the boundary conditions, one deduces that $\lim a_{n} G u_{n}=a G u$ weakly in $H_{1}$.

Let $v \in \operatorname{dom}(G)$. If $n \in \mathbb{N}$, then (12) gives

$$
\left(a_{n} G u_{n}, G v\right)_{H_{1}}+\left(m_{n} u_{n}, v\right)_{H_{0}}+\lambda\left(j\left(u_{n}\right), j(v)\right)_{H}=\left(\psi_{n}, j(v)\right)_{H} .
$$

Taking the limit $n \rightarrow \infty$, one establishes

$$
(a G u, G v)_{H_{1}}+(m u, v)_{H_{0}}+\lambda(j(u), j(v))_{H}=(\psi, j(v))_{H} .
$$

So $\mathfrak{b}(u, v)+\lambda(j(u), j(v))_{H}=(\psi, j(v))_{H}$. Therefore, $j(u) \in \operatorname{dom}\left(\Lambda_{H}\right)$ and one establishes that $\left(\lambda I+\Lambda_{H}\right) j(u)=\psi$. With the usual subsequence argument, we proved that $\lim (\lambda I+$ $\left.\Lambda_{H}^{(n)}\right)^{-1} \psi_{n}=\left(\lambda I+\Lambda_{H}\right)^{-1} \psi$ weakly in $H$. Now, Statement (b) follows by choosing $\psi_{n}=\psi$ for all $n \in \mathbb{N}$.

'(c)'. Finally, suppose that $\kappa$ is compact. Then, also $j$ is compact. Let $\lambda>\omega$. Suppose $\lim \left(\lambda I+\Lambda_{H}^{(n)}\right)^{-1}=\left(\lambda I+\Lambda_{H}\right)^{-1}$ in $\mathcal{L}(H)$ is false. Passing to a subsequence if necessary, there exist $\delta>0$ and $\psi_{1}, \psi_{2}, \ldots \in H$ such that

$$
\left\|\left(\lambda I+\Lambda_{H}^{(n)}\right)^{-1} \psi_{n}-\left(\lambda I+\Lambda_{H}\right)^{-1} \psi_{n}\right\|_{H}>\delta\left\|\psi_{n}\right\|_{H}
$$

for all $n \in \mathbb{N}$. Without loss of generality, we may assume that $\left\|\psi_{n}\right\|_{H}=1$ for all $n \in \mathbb{N}$. Passing again to a subsequence if necessary, there exists a $\psi \in H$ such that $\lim \psi_{n}=\psi$ weakly in $H$. Let $u_{n} \in V\left(\mathfrak{b}_{n}\right)$ and $u \in \operatorname{dom}(G)$ be as in Part (b) for all $n \in \mathbb{N}$. Then, $\lim u_{n}=u$ weakly in $\operatorname{dom}(G)$, so

$$
\lim _{n \rightarrow \infty}\left(\lambda I+\Lambda_{H}^{(n)}\right)^{-1} \psi_{n}=\lim _{n \rightarrow \infty} j\left(u_{n}\right)=j(u)=\left(\lambda I+\Lambda_{H}\right)^{-1} \psi
$$

in $H$ by the compactness of $j$. Similarly $\lim _{n \rightarrow \infty}\left(\lambda I+\Lambda_{H}^{(n)}\right)^{-1} \psi=\left(\lambda I+\Lambda_{H}\right)^{-1} \psi$ in $H$. So

$$
\lim _{n \rightarrow \infty}\left\|\left(\lambda I+\Lambda_{H}^{(n)}\right)^{-1} \psi_{n}-\left(\lambda I+\Lambda_{H}\right)^{-1} \psi_{n}\right\|_{H}=0 .
$$

This is a contradiction.

Note that the limit Dirichlet-to-Neumann graph $\Lambda_{H}$ is an operator in Theorem 6.4. In [4] Theorem 5.11, a different condition on the $a_{n}$ is used to obtain resolvent convergence for symmetric operators/graphs, but possibly multi-valued limit graph $\Lambda_{H}$. Since we do not wish to require symmetry in Theorem 6.4 and we need that the limit graph $\Lambda_{H}$ is m-sectorial, we require conveniently that all graphs are single-valued. See also the discussion at the end of Sect. 5.

Finally, we compare various conditions on the $a_{n}$ in the classical case.

Example 6.7 In this example, we characterize the condition $\lim _{n \rightarrow \infty}\left(\iota^{*} a_{n} \iota\right)^{-1}=\left(\iota^{*} a \iota\right)^{-1}$ in the weak operator topology of $\mathcal{L}(\operatorname{ran}(G))$ in Theorem 4.2 for the classical case of Example 2.3 and real symmetric coefficients.

Let $\Omega \subset \mathbb{R}^{d}$ be open, bounded and connected. Assume that $H^{1}(\Omega)$ embeds compactly into $L_{2}(\Omega)$. Further, let $H_{0}, H_{1}, G$ and $D$ be as in Example 2.3. We identify an element of $L_{\infty}\left(\Omega, \mathbb{C}^{d \times d}\right)$ with an element of $\mathcal{L}\left(H_{1}\right)$ in the natural way. Let $a, a_{1}, a_{2}, \ldots \in$ 
$L_{\infty}\left(\Omega, \mathbb{R}^{d \times d}\right)$. Suppose that $a_{n}=a_{n}^{*} \geqslant \mu I$ for all $n \in \mathbb{N}$ and $\sup _{n}\left\|a_{n}\right\|_{\mathcal{L}\left(H_{1}\right)}<\infty$. Moreover, assume that $a=a^{*} \geqslant \mu \mathrm{I}$. We emphasize that the $a_{n}$ and $a$ are real valued and symmetric. Then, the following three conditions are equivalent.

(a) The sequence $\left(a_{n}\right)_{n \in \mathbb{N}}$ is $H$-convergent to $a$.

(b) The sequence $\left(a_{n}\right)_{n \in \mathbb{N}}$ converges to $a$ independent of the boundary conditions.

(c) $\lim _{n \rightarrow \infty}\left(\iota^{*} a_{n} \iota\right)^{-1}=\left(\iota^{*} a \iota\right)^{-1}$ in the weak operator topology of $\mathcal{L}(\operatorname{ran}(G))$.

We proved the implications $(\mathrm{a}) \Rightarrow(\mathrm{b}) \Rightarrow$ (c) in Example 6.1 and Proposition 6.2. So it remains to show the implication (c) $\Rightarrow$ (a).

Suppose that $\lim _{n \rightarrow \infty}\left(\iota^{*} a_{n} \iota\right)^{-1}=\left(\iota^{*} a \iota\right)^{-1}$ in the weak operator topology of $\mathcal{L}(\operatorname{ran}(G))$. Since $a_{n} \geqslant \mu I$ for all $n \in \mathbb{N}$ and $\sup _{n}\left\|a_{n}\right\|_{\mathcal{L}\left(H_{1}\right)}<\infty$, it follows from [15] Theorem 6.5 that the sequence $\left(a_{n}\right)_{n \in \mathbb{N}}$ has a subsequence $\left(a_{n_{k}}\right)_{k \in \mathbb{N}}$ which is $H$-convergent. Hence, there exist $b \in L_{\infty}\left(\Omega, \mathbb{R}^{d \times d}\right)$ and $v>0$ such that $\operatorname{Re} b \geqslant v I$ and the sequence $\left(a_{n_{k}}\right)_{k \in \mathbb{N}}$ is $H$ convergent to $b$. Then, $b=b^{*}$ by [15] Lemma 10.2. It follows from the implication (a) $\Rightarrow$ (c) that $\lim _{k \rightarrow \infty}\left(\iota^{*} a_{n_{k}} \iota\right)^{-1}=\left(\iota^{*} b \iota\right)^{-1}$ in the weak operator topology of $\mathcal{L}(\operatorname{ran}(G))$. Therefore, $\left(\iota^{*} a \iota\right)^{-1}=\left(\iota^{*} b \iota\right)^{-1}$ and $\left(\iota^{*} a \iota\right)=\left(\iota^{*} b \iota\right)$. So

$$
(a \operatorname{grad} u, \operatorname{grad} v)_{L_{2}(\Omega)^{d}}=(b \operatorname{grad} u, \operatorname{grad} v)_{L_{2}(\Omega)^{d}}
$$

for all $u, v \in H^{1}(\Omega)$. Write $c=a-b$. Then, $(c \operatorname{grad} u, \operatorname{grad} v)_{L_{2}(\Omega)^{d}}=0$ for all $u, v \in$ $H^{1}(\Omega)$. We shall show that $c=0$. Let $\tau \in C_{c}^{\infty}(\Omega)$ and $\xi \in \mathbb{R}^{d}$. For all $\lambda \geqslant 1$, define $u_{\lambda} \in C_{c}^{\infty}(\Omega)$ by $u_{\lambda}(x)=\tau(x) e^{i \lambda \xi \cdot x}$. Then,

$$
0=\left(c \operatorname{grad} u_{\lambda}, \operatorname{grad} u_{\lambda}\right)_{L_{2}(\Omega)^{d}}=(c(\operatorname{grad} \tau+i \lambda \tau \xi),(\operatorname{grad} \tau+i \lambda \tau \xi))_{L_{2}(\Omega)^{d}} .
$$

Dividing by $\lambda^{2}$ and taking the limit $\lambda \rightarrow \infty$ gives $\int_{\Omega}|\tau(x)|^{2}\langle c(x) \xi, \xi\rangle_{\mathbb{C}^{d}} d x=0$. This implies that $\langle c(x) \xi, \xi\rangle_{\mathbb{C}^{d}}=0$ for almost all $x \in \Omega$. Since $\mathbb{R}^{d}$ is separable and $c=c^{*}$, this implies that $c=0$ almost everywhere. So $b=a$ almost everywhere. We proved that the sequence $\left(a_{n_{k}}\right)_{k \in \mathbb{N}}$ is $H$-convergent to $a$.

It follows similarly that every subsequence of $\left(a_{n}\right)_{n \in \mathbb{N}}$ has a subsubsequence which is $H$-convergent to $a$. Since the topology of $H$-convergence is metrizable by the discussion after Definition 6.4 in [15] one concludes that the sequence $\left(a_{n}\right)_{n \in \mathbb{N}}$ is $H$-convergent to $a$. This completes the proof of the implication (c) $\Rightarrow(\mathrm{a})$.

In [19] Theorem 1.2, it is proved that the three equivalent conditions are also equivalent to a version of Condition (c), where grad is replaced by grad and $\iota$ by the embedding ran (grad) $\subset$ $H_{1}$; see also Remark 6.3.

Remark 6.8 In the situation of Example 6.7, we deduce that convergence of $\left(a_{n}\right)_{n \in \mathbb{N}}$ in the weak* topology of $L_{\infty}\left(\Omega, \mathbb{R}^{d \times d}\right)$ neither implies nor is implied by $\lim \left(\iota^{*} a_{n} \iota\right)^{-1}=\left(\iota^{*} a \iota\right)^{-1}$ in the weak operator topology of $\mathcal{L}(\operatorname{ran}(G))$. One may also consult [20, Examples 3.2 and 3.4] on this.

\section{More examples}

The first example is from linearized elasticity.

Example 7.1 Let $\Omega \subset \mathbb{R}^{d}$ be open. Set

$$
L_{2, \operatorname{sym}}(\Omega)=\left\{S \in L_{2}(\Omega)^{d \times d}: S^{T}=S \text { a.e. }\right\} .
$$


Choose $H_{0}=L_{2}(\Omega)^{d}$ and $H_{1}=L_{2, \operatorname{sym}}(\Omega)$. Define $\widehat{G}: C_{c}^{\infty}(\Omega)^{d} \rightarrow L_{2, \operatorname{sym}}(\Omega)$ by

$$
(\widehat{G} u)_{k l}=\frac{1}{2}\left(\partial_{k} u_{l}+\partial_{l} u_{k}\right) .
$$

Further define $\widehat{D}: C_{c}^{\infty}(\Omega)^{d \times d} \cap L_{2, \operatorname{sym}}(\Omega) \rightarrow L_{2}(\Omega)^{d}$ by

$$
(\widehat{D} q)_{k}=\sum_{l=1}^{d} \partial_{l} q_{k l} .
$$

Then, $\operatorname{dom}(\widehat{G})$ is dense in $H_{0}$ and $\operatorname{dom}(\widehat{D})$ is dense in $H_{1}$. Moreover, using integration by parts one deduces that (2) is valid. Then, one can apply Example 2.2.

Korn's first inequality implies that $\left\|\partial_{k} u_{l}\right\|_{L_{2}(\Omega)} \leqslant \sqrt{2}\|\widehat{G} u\|_{H_{1}}$ for all $u \in C_{c}^{\infty}(\Omega)^{d}$ and $k, l \in\{1, \ldots, d\}$. So $\operatorname{dom}(\stackrel{\circ}{G}) \subset H_{0}^{1}(\Omega)$. In particular, the inclusion $\operatorname{dom}(\stackrel{\circ}{G}) \subset H_{0}$ is compact if $\Omega$ is bounded.

Under some regularity conditions on the boundary of $\Omega$, Korn's second inequality states that there exists a $c>0$ such that $\left\|\partial_{k} u_{l}\right\|_{L_{2}(\Omega)} \leqslant c\|u\|_{\operatorname{dom}(G)}$ for all $u \in \operatorname{dom}(G)$ and $k, l \in\{1, \ldots, d\}$. For example, if $\Omega$ is bounded with a Lipschitz boundary, then Korn's second inequality is valid. For an easy proof see [10] Section 3. If Korn's second inequality is valid, then $\operatorname{dom}(G) \subset H^{1}(\Omega)^{d}$. Consequently, if Korn's second inequality is valid and $\Omega$ has a continuous boundary, then the inclusion $H^{1}(\Omega) \subset L_{2}(\Omega)$ is compact and hence the inclusion $\operatorname{dom}(G) \subset H_{0}$ is compact. We point out that Korn's second inequality is not a necessary condition for the inclusion $\operatorname{dom}(G) \subset H_{0}$ to be compact, see [21] Theorem 1 .

In particular, suppose $\Omega$ is bounded with a Lipschitz boundary and write $\Gamma=\partial \Omega$. Let $\sigma \in\left(-\infty, \frac{1}{2}\right]$ and set $H=H^{\sigma}(\Gamma)^{d}$. Then, $\operatorname{Tr} u \in H$ for all $u \in \operatorname{dom}(G)$. Moreover, $\left.\operatorname{Tr}\right|_{\mathrm{BD}(G)}: \operatorname{BD}(G) \rightarrow H$ is injective and has dense range. So one can consider as in Sect. 3 a Dirichlet-to-Neumann operator in $H$. Note that $\left.\operatorname{Tr}\right|_{\mathrm{BD}(G)}$ is compact if $\sigma<\frac{1}{2}$.

The second example is from electro-magneto statics.

Example 7.2 Let $\Omega \subset \mathbb{R}^{3}$ be open. Using integration by parts, one deduces that

$$
(\operatorname{curl} u, v)_{L_{2}(\Omega)^{3}}=(u, \operatorname{curl} v)_{L_{2}(\Omega)^{3}}
$$

for all $u, v \in C_{c}^{\infty}(\Omega)^{3}$. Therefore, let $H_{0}=H_{1}=L_{2}(\Omega)^{3}$ and define $\widehat{G}=\widehat{D}: C_{c}^{\infty}(\Omega)^{3} \rightarrow$ $L_{2}(\Omega)^{3}$ by $\widehat{G} u=\widehat{D} u=i$ curl $u$. Then, (2) is satisfied. Using the construction in Example 2.2, one obtains a new example.

Acknowledgements The authors wish to thank the referee for raising questions which improved the paper. The third named author expresses his gratitude for the wonderful atmosphere and hospitality extended to him during a two-month research visit at the University of Auckland. Part of this work is supported by the Marsden Fund Council from Government funding, administered by the Royal Society of New Zealand. Part of this work is supported by the EU Marie Curie IRSES program, Project AOS, No. 318910. Part of this work was carried out with financial support of the EPSRC grant EP/L018802/2: Mathematical foundations of metamaterials: homogenization, dissipation and operator theory.

\section{References}

1. Arendt, W., Chill, R., Seifert, C., Vogt, H., Voigt, J.: Internet Seminar 18 (2015). https://www.mat.tuhh. de/veranstaltungen/isem18/pdf/LectureNotes.pdf

2. Arendt, W., ter Elst, A.F.M.: The Dirichlet-to-Neumann operator on rough domains. J. Differ. Equ. 251, 2100-2124 (2011) 
3. Arendt, W., ter Elst, A.F.M.: Sectorial forms and degenerate differential operators. J. Oper. Theory 67, 33-72 (2012)

4. Arendt, W., ter Elst, A.F.M., Kennedy, J.B., Sauter, M.: The Dirichlet-to-Neumann operator via hidden compactness. J. Funct. Anal. 266, 1757-1786 (2014)

5. Axelsson, A., Keith, S., McIntosh, A.: Quadratic estimates and functional calculi of perturbed Dirac operators. Invent. Math. 163, 455-497 (2006)

6. Behrndt, J., ter Elst, A.F.M.: Dirichlet-to-Neumann maps on bounded Lipschitz domains. J. Differ. Equ. 259, 5903-5926 (2015)

7. Behrndt, J., ter Elst, A.F.M.: The Dirichlet-to-Neumann map for Schrödinger operators with complex potentials. Discrete Contin. Dyn. Syst. Ser. S 10, 661-671 (2017)

8. McLean, W.: Strongly Elliptic Systems and Boundary Integral Equations. Cambridge University Press, Cambridge (2000)

9. Murat, F., Tartar, L.: $H$-convergence. In: Cherkaev, A., Kohn, R. (eds.) Topics in the Mathematical Modelling of Composite Materials, Programming of Nonlinear Differential Equations Application, vol. 31, pp. 21-43. Birkhäuser, Boston (1997)

10. Nitsche, J.A.: On Korn's second inequality. RAIRO Anal. Numér. 15, 237-248 (1981)

11. Picard, R., Seidler, S., Trostorff, S., Waurick, M.: On abstract div-grad systems. J. Differ. Equ. 260, 4888-4917 (2016)

12. Picard, R., Trostorff, S., Waurick, M.: On a class of boundary control problems. Oper. Matrices 8, 185-204 (2014)

13. Picard, R., Trostorff, S., Waurick, M.: On a comprehensive class of linear control problems. IMA J. Math. Control Inf. 33, 257-291 (2016)

14. Rondi, L.: Continuity properties of Neumann-to-Dirichlet maps with respect to the $H$-convergence of the coefficients matrices. Inverse Probl. 31, 045002 (2015)

15. Tartar, L.: The General Theory of Homogenization. A Personalized Introduction. Notes of the Unione Matematica Italiana. Springer, Berlin (2009)

16. Trostorff, S.: A characterization of boundary conditions yielding maximal monotone operators. J. Funct. Anal. 267, 2787-2822 (2014)

17. Trostorff, S., Waurick, M.: A note on elliptic type boundary value problems with maximal monotone relations. Math. Nachr. 287, 1545-1558 (2014)

18. Waurick, M.: On the continuous dependence on the coefficients of evolutionary equations. Habilitation thesis, TU Dresden (2016)

19. Waurick, M.: $G$-convergence and the weak operator topology. Proc. Appl. Math. Mech. 16, 883-884 (2016)

20. Waurick, M.: Nonlocal $H$-convergence. arXiv-preprint arXiv:1804.02026 (2018)

21. Weck, N.: Local compactness for linear elasticity in irregular domains. Math. Methods Appl. Sci. 17, 107-113 (1994) 